\title{
Schriftliches mathematisches Argumentieren in zwei unterschiedlichen Inhaltsbereichen in den Jahrgangsstufen 4-6
}

\author{
Esther Brunner $(\mathbb{D} \cdot$ Jonas Lampart • Romaine Jullier
}

Eingegangen: 4. Juni 2020 / Angenommen: 21. Januar 2022 / Online publiziert: 17. Februar 2022

(C) Der/die Autor(en) 2022

Zusammenfassung Im Rahmen einer Studie zum schriftlichen mathematischen Argumentieren wurde untersucht, wie Schülerinnen und Schüler der Jahrgangsstufen 4-6 $(N=866)$ beim Lösen von zwei Aufgaben aus unterschiedlichen Inhaltsbereichen - Arithmetik und Geometrie - argumentieren. Dazu wurden zwei Aufgaben entlang verschiedener theoretisch hergeleiteter Teilkompetenzen mathematischen Argumentierens entwickelt und in Teilaufgaben strukturiert. Zudem wurde geprüft, ob sich Unterschiede zwischen den drei Jahrgangsgruppen nachweisen lassen. Um die Bearbeitung der Begründungsaufgaben bezüglich zentraler inhaltlicher Merkmale beschreiben zu können, wurde entlang theoretischer Grundlagen ein Kategoriensystem entwickelt, das sowohl verschiedene Teilkompetenzen mathematischen Argumentierens in den Blick nimmt als auch die von den Schülerinnen und Schülern genutzten inhaltlichen Ideen erfasst und diese Bearbeitungsmerkmale jeweils bezüglich ihrer Ausführungsqualität einschätzt. Bei der Anwendung des Kategoriensystems ergab sich eine zufriedenstellende bis gute Interraterreliabilität. Vorgestellt werden Ergebnisse zu gezeigten Teilkompetenzen mathematischen Argumentierens sowie einbezogenen inhaltlichen Ideen für das Lösen der beiden Aufgaben. Die Befunde legen nahe, dass der Inhaltsbereich für die Bearbeitung in verschiedener Hinsicht eine Rolle spielt und dass sich die drei Jahrgangsstufen bezüglich verschiedener Merkmale der Bearbeitung voneinander unterscheiden.

Esther Brunner $(\bowtie) \cdot$ Jonas Lampart

Pädagogische Hochschule Thurgau, Unterer Schulweg 3, 8280 Kreuzlingen, Schweiz

E-Mail: esther.brunner@phtg.ch

Jonas Lampart

E-Mail: jonas.lampart@phtg.ch

Romaine Jullier

Pädagogische Hochschule Zürich, Lagerstr. 2, 8090 Zürich, Schweiz

E-Mail: romaine.jullier@phzh.ch 
Schlüsselwörter Mathematikdidaktik · Mathematisches Argumentieren ·

Schriftliches Begründen · Leistung · Primarstufe

Mathematics Education Subject Classification 97D10 · 97D60 - 97E50

\title{
Written Mathematical Reasoning in Two Different Content Areas in Grades 4-6
}

\begin{abstract}
In the context of a study on written mathematical reasoning, we investigated how students in grades $4-6(N=866)$ deal with two reasoning problems from different content areas (arithmetic and geometry). Moreover, we examined whether there are differences between the three age groups. So as to be able to describe the children's reasoning skills comprehensively and with regard to central contentrelated features, we developed a category system by means of an approach that referred to theoretical considerations. The category system covers a broad range of mathematical reasoning skills as well as the content-related ideas that the children came up with and assesses these features in terms of their quality of application. The coding process resulted in satisfactory to good interrater reliability. In our paper, we present our findings both concerning the children's demonstration of different mathematical reasoning skills and concerning their content-related ideas for solving the two reasoning problems. Our findings suggest that the content area plays a role with regard to the reasoning skills in various respects and that the three grades differ systematically from each other in several features of the reasoning process.
\end{abstract}

Keywords Mathematics education · Mathematical argumentation · Written reasoning $\cdot$ Student achievement $\cdot$ Primary school

\section{Einleitung}

Mathematisches Argumentieren gehört zu den zentralen, während der obligatorischen Bildung von allen Lernenden zu erwerbenden mathematischen Kompetenzen. Bildungspläne und Kompetenzbeschreibungen (z. B. D-EDK 2016; KMK 2005; Walther et al. 2008) oder Aufgabenbeispiele aus standardisierten Tests, wie beispielsweise VERA 3 (IQB 2013), geben Anhaltspunkte in Bezug darauf, welche Kompetenzen zu einem bestimmten Zeitpunkt erwartet werden. Sie beschreiben einen Kompetenzaufbau über die verschiedenen Schulstufen hinweg (z. B. D-EDK 2016; KMK 2005; KMK und IQB 2017) oder definieren inhaltliche Anforderungsniveaus in Bezug auf mathematisches Argumentieren (Bezold 2009; Leiss und Blum 2006), deren Erreichung im Rahmen des Bildungsmonitorings mittels standardisierter Tests punktuell überprüft werden kann. Während für die Sekundarstufe mittlerweile zahlreiche und differenzierte Forschungsbefunde zur Frage verfügbar sind, über welche Argumentierkompetenzen Lernende in diesem Alter bereits verfügen, liegen bislang erst wenige empirische Befunde vor (z.B. für Jahrgangsstufe 3: Bezold 2009; für 
die Jahrgangsstufen 3, 4 und 6: Neumann et al. 2014), die vertieft klären würden, was Lernende der Primarstufe ${ }^{1} \mathrm{zu}$ einem bestimmten Zeitpunkt tatsächlich können.

Betrachtet man die Forschungsliteratur zur Primarstufe, lässt sich feststellen, dass zwar zahlreiche aufschlussreiche Fallstudien insbesondere zu mündlichem Argumentieren vorliegen, aber insgesamt erst wenig gesichertes Wissen zu selbstständigem schriftlichem Begründen von Lernenden vorhanden ist. Bislang fehlen insbesondere breite Forschungserkenntnisse, die hilfreich sind, um mathematische Argumentationsprozesse von Lernenden im Primarschulalter differenziert beschreiben und sie auf der Grundlage einer solchen - inhaltsbereichsübergreifend charakterisierten - Beschreibung von Teilkompetenzen über verschiedene Inhaltsbereiche hinweg analysieren zu können, um darauf basierend auch (quasi-)längsschnittliche Entwicklungen in den Blick nehmen zu können. Hier setzt die vorliegende Studie an.

\section{Theoretische Grundlagen}

\subsection{Begriffsklärung}

Die Begriffe „Argumentieren“, „Begründen“ und „Beweisen“ werden in der Literatur unterschiedlich definiert und verwendet. Die in diesem Zusammenhang geführte Kontroverse konzentriert sich insbesondere auf die Beziehung zwischen Argumentieren und Beweisen, die von den einen als Relation zwischen zwei klar voneinander abgrenzbaren mathematischen Prozessen betrachtet wird (vgl. z. B. Balacheff 1988; Duval 1991), während andere das Verhältnis eher innerhalb eines Kontinuums ansiedeln (Boero et al. 2010; Pedemonte 2007). Als Konsens gilt, dass beim Argumentieren, Begründen und Beweisen ,Aussagen auf Gründe zurückgeführt werden sollen“ (Jahnke und Ufer 2015, S. 333). Welcher Art die Gründe sind, die in diesem Prozess als akzeptabel betrachtet werden, ist abhängig vom Lernstand und dem Entwicklungsniveau der Lernenden (z. B. Hanna 1997; Jahnke und Ufer 2015). Zwingend ist in sämtlichen Fällen, dass die gezogenen Schlüsse logisch gültig sind und auf wahren Aussagen beruhen. Diese grundlegende Unterscheidung zwischen Wahrheit und Gültigkeit (Durand-Guerrier 2008) verdeutlicht die Wichtigkeit der logischen Struktur einer Begründung, d.h. der formalen Beziehung zwischen den Prämissen und der Konklusion, und derjenigen des Zutreffens, d.h. der Korrektheit der Inhalte. Dieser inhaltliche Aspekt wird auch als „Anchoring“ (Lithner 2008) innerhalb des (vorhandenen) mathematischen Wissens bezeichnet. Im Fokus des „Anchorings“ stehen zum einen diejenigen Wissensbestandteile, auf die beim Argumentieren zurückgegriffen wird. Zum anderen geht es um die Frage, welche neuen, inhaltsbezogenen Verknüpfungen im Sinne von inhaltlichen Ideen hergestellt werden können.

Der Begriff des Begründens im Sinne von „Reasoning“ wird oft als Oberbegriff für verschiedene Arten des Begründens verwendet und umfasst sowohl Formen des Argumentierens als auch Formen des Beweisens als besondere Varietät mathema-

\footnotetext{
1 Unter Primarstufe werden - wie in der Schweiz üblich - die Jahrgangsstufen 1-6 verstanden.
} 
tischen Begründens (Brunner 2014). Gleichzeitig ist „Begründen“ aber nicht nur ein Sammelbegriff für verschiedene Arten des Angebens mathematischer Gründe, sondern auch ein bestimmter Schritt innerhalb des gesamten Prozesses mathematischen Argumentierens, dem andere Handlungen vorausgehen oder ggf. folgen. Die deutschen Bildungsstandards (KMK 2005) lösen dieses terminologische Kategorienproblem durch Verwendung der Bezeichnung „mathematisches Argumentieren“ für den Gesamtprozess und die Spezifikation der einzelnen Teilprozesse durch die Benennung von konkreten Handlungen wie „Begründung suchen“. Wir lehnen uns an diese Terminologie an und sprechen im Rahmen dieses Beitrags von ,,mathematischem Argumentieren“ im Sinne von „Reasoning“ als umfassendem Prozess und als Oberbegriff für verschiedene Arten von Begründen und verstehen ihn analog $\mathrm{zu}$,mathematisches Argumentieren“ in den deutschen Bildungsstandards, während sich „Begründen“ im engen Sinne auf „Begründung angeben“, d.h. den Begründungsschritt bezieht.

\subsection{Aktivitäten mathematischen Argumentierens}

„Mathematisches Argumentieren“als Terminus und eine der zentralen Kompetenzen wird in den deutschen Bildungsstandards nur knapp und als „Oberbegriff“ für die folgenden drei „Punkte“ umschrieben (Walther et al. 2008, S. 32): „mathematische Aussagen hinterfragen und auf Korrektheit prüfen“, „mathematische Zusammenhänge erkennen und Vermutungen entwickeln“ sowie „Begründungen suchen und nachvollziehen“.

Eine solche Umschreibung legt ein weites Verständnis von mathematischem Argumentieren (Reid und Knipping 2010) nahe, das aus verschiedenen Handlungen und Teilkompetenzen besteht und sich nicht auf die Konstruktion einer Begründung beschränkt. Basis dieser Handlungen sind experimentelle bzw. heuristische Phasen, d.h. Phasen des Erkundens und Ausprobierens (Jahnke und Ufer 2015).

Jeannotte und Kieran (2017, S. 9 ff.) beschreiben auf der Grundlage einer Literaturrecherche folgende „Prozessaspekte“ mathematischen Argumentierens und ordnen sie drei unterschiedlichen Arten von Prozessen zu: 1) fünf „Prozesse“ zum Suchen von Ähnlichkeiten und Unterschieden: Generalisieren, Vermuten, Identifizieren von Mustern, Vergleichen, Klassifizieren; 2) vier „Prozesse“ mit Bezug zur Validierung: Validieren, Rechtfertigen, Beweisen, formales Beweisen und 3) einen „Prozess“ zur Unterstützung für andere Argumentationsprozesse: Exemplifizieren. Allerdings ist kritisch anzumerken, dass Validierungsaspekte sämtliche andere Prozesse begleiten, d.h. wiederholt werden müssen und sich somit kategoriell von den anderen genannten Prozessen unterscheiden. Ebenfalls kritisch zu fragen ist, inwiefern das Generalisieren tatsächlich den Prozessen zum Suchen von Ähnlichkeiten und Unterschieden zugeordnet werden soll. Jeannotte und Kieran (2017, S. 9) definieren Generalisieren als ,,process that infers narratives about a set of mathematical objects or a relation between objects of the set from a subset of this set". Generalisieren bezieht sich somit auf eine Konklusion und Verallgemeinerung. Im Zusammenhang mit Prozessen zum Suchen von Ähnlichkeiten und Unterschieden geht es aber weniger um das Herstellen einer Konklusion als um das Erkennen und Herausarbeiten eines übergreifenden Musters. 
Auch Neumann et al. (2014) befassen sich mit dem Prozess des mathematischen Argumentierens und zwar insbesondere auch aus einer sprachlichen Perspektive heraus, da sie sowohl den Beweis als auch die Begründung als ,hochgradig sprachlich verankert" (S. 114) interpretieren und auf den schriftsprachlichen Ausdruck mathematischer Begründungskompetenz fokussieren. Für sie ist „mathematisches Begründen ... wie das Beweisen eine Tätigkeit, durch welche der Wahrheitsgehalt einer Aussage geprüft und nachgewiesen bzw. widerlegt wird“, wobei sie ,mathematisches Begründen“"als einen bestimmten Schritt innerhalb des Handlungsmusters „mathematisches Argumentieren“ auffassen, das die folgenden vier Schritte beinhaltet: „Entdecken, Beschreiben, Hinterfragen und Begründen mathematischer Gesetzmäßigkeiten und Zusammenhänge" (S. 114). Auch hier ist - wie bei Jeannotte und Kieran (2017) - kritisch anzumerken, dass sich der Schritt des Hinterfragens kategoriell von den anderen drei unterscheidet, weil er wiederholt bei allen anderen Schritten angewendet werden muss.

Vergleicht man diese „Schritte“ mit den „Prozessen“, wie sie von Jeannotte und Kieran (2017) aufgelistet werden, zeigen sich Gemeinsamkeiten und Unterschiede. Der erste Schritt bei Neumann et al. (2014, S. 114) ist vergleichbar mit den Prozessen zum Suchen von Ähnlichkeiten und Unterschieden bei Jeannotte und Kieran (2017). Der zweite Schritt hingegen, das Beschreiben, der bei Jeannotte und Kieran (2017) fehlt, nimmt im Zusammenhang mit der entdeckten, d.h. identifizierten mathematischen Struktur eine Brückenfunktion zwischen Identifizieren und Begründen ein. Einzelne Prozesse des Validierens korrespondieren mit dem dritten Schritt bei Neumann et al. (2014, S. 114), dem Hinterfragen. Andere hingegen lassen sich eher dem vierten Schritt, dem Begründen, zuordnen. Der Prozess des Verallgemeinerns als eigenständiger Prozess hingegen fehlt bei Neumann et al. (2014, S. 114) und wird als Teil einer Begründung verstanden. Aus einer kognitiven Perspektive heraus kann Verallgemeinern aber als eine weitere Abstraktionsstufe interpretiert werden, weil es bedeutet, ,von der Betrachtung eines gegebenen Aggregats von Objekten zu der eines großen Aggregats“ überzugehen (Polya 1954, S. 33).

Für den Primarbereich lassen sich vor diesem Hintergrund und ausgehend von Erkundung und Exploration zumindest vier relevante Teilkompetenzen mathematischen Argumentierens als inhaltsübergreifende, spezifische Handlungen beschreiben (Brunner 2019a; Lindmeier et al. 2018): (1) Erkennen/Identifizieren einer bestimmten mathematischen Struktur mit den diese konstituierenden Merkmalen, (2) $\mathrm{Be}$ schreiben/Repräsentieren der erkannten Struktur mitsamt der sie konstituierenden Merkmalen, (3) Entwickeln und Formulieren von Begründungen für das Zustandekommen der Struktur und (4) Verallgemeinern und damit der Schluss aus dem Einzelfall auf das Allgemeine. Die Begründung für das Zustandekommen einer bestimmten Struktur oder die Geltung einer Behauptung beinhaltet den Einbezug einer inhaltlichen Idee (Brunner et al. 2020) und verlangt daher die Berücksichtigung einer breiteren mathematischen Wissensbasis, und somit ein „Anchoring“ (Lithner 2008). Diese inhaltliche Idee wird meist bereits bei der Beschreibung/Repräsentation der erkannten Struktur deutlich und bildet auch die inhaltliche Grundlage des ,Anchorings" für das Verallgemeinern, das darauf abzielt, Einsicht in Bezug darauf zu erlangen, weshalb ein postulierter Sachverhalt oder eine Vermutung notwendigerweise immer gelten muss. Dies stellt die Voraussetzung dar, um prognostische 
Annahmen für weitere Fälle treffen zu können, die anschließend validiert bzw. falsifiziert werden. Begründen und Verallgemeinern werden begleitet durch Handlungen des Evaluierens und Prüfens einer Aussage bezüglich ihrer Gültigkeit, im Fall des Begründens der lokalen Gültigkeit und im Fall des Verallgemeinerns der universellen Gültigkeit. Dabei muss sichergestellt werden, dass nur auf gesicherte Aussagen zurückgegriffen wird und dass ausschließlich - von der mathematischen Community bzw. von der Lerngemeinschaft - akzeptierte Schlussregeln befolgt werden.

Die Teilkompetenzen mathematischen Argumentierens können auch anhand von schriftlichen Dokumenten, in denen Lernende ihre Überlegungen festhalten, nachvollzogen werden. Das Erkennen (1) zentraler konstitutiver Merkmale kann daran festgemacht werden, dass Lernende beispielsweise ein Muster oder eine bestimmte mathematische Struktur weiterführen können. Dies ist nur dann möglich, wenn sie die entscheidenden Merkmale erkannt haben. Beschreiben/Repräsentieren ${ }^{2}$ (2) wird erkennbar, wenn Lernende beispielsweise in der Lage sind, die erkannte Struktur mit den sie konstituierenden Merkmalen in Worte zu fassen oder durch eine Skizze zu veranschaulichen, ohne aber ihr Zustandekommen zu begründen. Die Fähigkeit, Begründungen entwickeln und diese mithilfe situativ und altersgemäß angemessenen Mitteln ausdrücken zu können (3), zeigt sich in einer Begründung für das Zustandekommen eines bestimmten Sachverhaltes, die unterschiedlich repräsentiert sein kann (als Text, als Skizze oder formal-symbolisch). Ob es Lernenden darüber hinaus möglich ist, ihre Überlegungen zu verallgemeinern (4), lässt sich ebenfalls anhand ihrer schriftlich fixierten Begründung erkennen. Eine Verallgemeinerung wird erkennbar, wenn die Begründung über den Einzelfall hinausweist und zu klären versucht, warum etwas notwendigerweise immer gelten muss. Dies wird meist als Text oder formal-symbolisch repräsentiert.

Inwiefern es sich bei diesen vier Handlungen nicht nur um das mathematische Argumentieren strukturierende Aktivitäten, sondern auch um Entwicklungsschritte handelt, ist offen. Es wird daher nicht von Schritten gesprochen, wie dies Neumann et al. (2014) tun, um den Eindruck eines Entwicklungsmodells zu vermeiden, sondern von Handlungen, die zum Prozess des mathematischen Argumentierens gehören und die im Rahmen dieser Studie als Teilkompetenzen interpretiert werden.

\subsection{Zum Forschungsstand schriftlichen mathematischen Argumentierens in der Primarschule}

\subsubsection{Beschreibung von Teilkompetenzen mathematischen Argumentierens}

Bislang liegen kaum Studien vor, die eine Folge einzelner Handlungen in den Blick nahmen und systematisch Teilkompetenzen von Lernenden beim mathematischen Argumentieren untersuchten. Verschiedene empirische Arbeiten zeigten auf, wo Schwierigkeiten auch älterer Lernender generell und im Besonderen beim Schließen auftreten (z. B. Heinze 2004; Reiss et al. 2006), und legen somit nahe, dass der Begründungsschritt als besonders fehleranfällig und schwierig zu betrachten ist. Dieser Befund ist mittlerweile solide erhärtet (z. B. English 2004; Galotti et al. 1997). Zum

\footnotetext{
2 Im Folgenden kurz als „Beschreiben“ aufgeführt.
} 
Verallgemeinern wiederum zeigten verschiedene Studien auf, dass beispielgebundenes Beweisen bereits bei Lernenden des sechsten Schuljahres (Goldberg 1988) und auch bei Lernenden der Grundschule ${ }^{3}$ (Krumsdorf 2009) erfolgreich verlaufen kann. Das Vornehmen von Verallgemeinerungen beim deduktiven Beweisen hingegen stellt nicht nur für Primarschulkinder, sondern auch für ältere Lernende der Klassen 7-13 eine große Herausforderung dar (z. B. Heinze 2004). Neumann et al. (2014) zeigten in ihrer Studie, dass sich das Entdecken und Fortführen mathematischer Zusammenhänge für Lernende der Jahrgangsstufen 3, 4 und 6 einfacher gestalteten als das Verknüpfen mit einer mathematischen Begründungsstruktur und dass ihnen diese beiden Handlungen auch leichter fielen als das Verbalisieren der erkannten Begründungsstruktur. Diesen Befunden zufolge wäre zu vermuten, dass Erkennen und Beschreiben relativ leicht gelingen dürften, während Begründen und Verallgemeinern in der Regel Probleme bereiten.

\subsubsection{Untersuchungen in unterschiedlichen Inhaltsbereichen}

Viele der Befunde aus dem Bereich der Sekundarstufe ${ }^{4}$ stammen aus Studien, die sich mit geometrischen Kontexten befassten (z. B. Heinze und Reiss 2004; Ufer et al. 2009). Andere Studien fokussierten Beweiskompetenzen - beispielsweise für den Hochschulkontext - im Inhaltsbereich „Arithmetik/Algebra“ (z. B. Kempen 2019). Vereinzelt liegen Studien vor, bei denen eine Aufgabe aus dem Bereich elementarer Zahlentheorie mit arithmetisch-algebraischen bzw. mit geometrischen Mitteln bearbeitet werden sollte (z.B. Brunner 2013; Kempen 2019), was aber keinen Vergleich der Bearbeitung mit Blick auf unterschiedliche Inhaltsbereiche zulässt. Eine Studie von Tebaartz und Lengnink (2015) setzte zwar zwei Aufgaben aus unterschiedlichen Inhaltsbereichen ein, analysierte die Unterschiede zwischen den Jahrgangsstufen jedoch getrennt für die beiden Aufgaben bzw. Inhaltsbereiche und konzentrierte sich insbesondere auf die verwendeten Schlussweisen, die Korrektheit und die Vollständigkeit der Begründungen. Fokussierung auf einen einzigen Inhaltsbereich wurde auch in Studien aus der Primarstufe vorgenommen. Während sich einige davon auf mathematisches Argumentieren im Bereich der Arithmetik bezogen (z.B. Bezold 2009; Welsing 2017), befassten sich andere mit mathematischem Argumentieren in geometrischen (z.B. Schmid 2017; Unterhauser und Gasteiger 2017) oder in stochastischen Kontexten (z.B. Vogt 2021).

Nicht systematisch untersucht ist, ob sich mathematisches Argumentieren inhaltsbezogen unterschiedlich zeigt und ob es eine vom Inhalt unabhängige Kompetenz mathematischen Argumentierens gibt. Zieht man die Arbeiten aus dem Bereich der Gestaltpsychologie (z. B. Wertheimer 1964) oder die Arbeiten mit generischen Beispielen (z.B. Kempen 2013; Mason und Pimm 1984) heran, die sich oft auf ganz

\footnotetext{
3 Unter Grundschule werden die Jahrgangsstufen 1-4 verstanden.

4 Unter Sekundarstufe wird in Deutschland i.d. R. die Jahrgangsstufen ab Klasse 5 verstanden, in der Schweiz hingegen ab Klasse 7. Es wird hier von Sekundarstufe gesprochen, wenn der fokussierte Bereich auch die Jahrgangsstufen 7-9 berücksichtigen. Bezieht sich eine Studie ausschliesslich auf die Jahrgangsstufen 5-6, wird sie im Rahmen dieses Beitrags dem Bereich der Primarstufe zugerechnet, da hier unter Primarstufe die Jahrgangsstufen 1-6 gefasst werden.
} 
bestimmte Inhalte beziehen, bei denen sich Einsicht unmittelbar ablesbar erzeugen lässt, liegt die Vermutung nahe, dass die Leistung beim mathematischen Begründen je nach Inhaltsbereich unterschiedlich ausfallen dürfte. Notwendig sind daher Studien, die für dieselbe Population untersuchen, inwiefern sich bei der schriftlichen Bearbeitung von Begründungsaufgaben aus unterschiedlichen Inhaltsbereichen systematische Unterschiede feststellen lassen. Eine Klärung dieser Frage würde allerdings Aufgaben erfordern, die differente Inhaltsbereiche umfassen, aber ähnlich konstruiert sind, beispielsweise entlang ähnlicher Teilaufgaben. Different wäre nebst dem Inhaltsbereich aber auch die Gestaltung der Aufgabe und Teilaufgaben, da Aufgaben aus geometrischen Kontexten i.d. R. mit einer geometrischen Darstellung gestellt werden, solche aus arithmetischen Kontexten hingegen entsprechend eine arithmetische oder sprachliche Form aufweisen. In diesem Zusammenhang dürfte wohl davon auszugehen sein, dass insbesondere jüngeren Kindern das Begründen in geometrischen Kontexten aufgrund der unmittelbaren Anschaulichkeit (Blum und Kirsch 1991; Krauthausen 2001; Wertheimer 1964; Wittmann und Müller 1988), die zum Probieren anregt, leichter fallen dürfte als Begründen in arithmetischen Kontexten.

\subsubsection{Untersuchungen zu Argumentstruktur und inhaltichen Ideen}

Es liegen zahlreiche Untersuchungen vor, die mathematisches Argumentieren von Primarschülerinnen und -schülern interpretativ, rekonstruktiv, aus epistemologischer Perspektive oder entlang des Toulmin-Schemas der Struktur eines Arguments (Toulmin 1996) analysierten (z. B. Krummheuer 2007; Landgraf 2019; Schwarzkopf 2000, 2015; Stylianides 2016) und einen äußerst detaillierten Einblick in das Denken und Argumentieren der jeweiligen Kinder geben und zwar sowohl zu inhaltlichen wie strukturellen Aspekten. Diese aufwändigen Studien beziehen sich in der Regel auf relativ kleine Stichproben.

Studien, die eine größere Population in den Blick nahmen, konzentrierten sich beispielsweise auf die Prüfung der Korrektheit und der Vollständigkeit der inhaltlichen Stützen der Konklusion eines Arguments (z. B. Jablonski und Ludwig 2019). Dies ist auch in Studien zum schriftlichen Argumentieren verbreitet (z. B. Kempen 2019; Neumann et al. 2014; Tebaartz und Lengnink 2015). Inhaltliche Aspekte wurden im Zusammenhang mit der Bewertung der formulierten Begründung berücksichtigt und beispielsweise als Stufe innerhalb einer Ratingskala einbezogen (z. B. Neumann et al. 2014), aber eine systematische Erfassung von inhaltlichen Ideen als kreativem Beitrag zur Lösungsfindung und als „Anchoring“ (Lithner 2008) sowie als Ausdruck einer inhaltlichen Einsicht (Wertheimer 1964) und damit als Bezug zur notwendigen mathematischen Wissensbasis erfolgte bislang nur vereinzelt (z. B. Sumpter 2018). Die Erfassung einer inhaltlichen Idee mit Rückbezug auf die Vollständigkeit und die Korrektheit der angegebenen Gründe inkl. deren Stütze in einer geeigneten mathematischen Wissensbasis ist daher insbesondere bei größeren Stichproben noch nicht umfassend geleistet. 


\subsubsection{Längsschnittliche bzw. quasi-längsschnittliche Untersuchungen}

Es liegen bislang keine Ergebnisse zur Entwicklung schriftlichen Argumentierens in der Mathematik der Primarschule vor (Kosko und Zimmerman 2019). Gemäß dem aktuellen Forschungsstand ist davon auszugehen, dass mit zunehmendem Alter elaboriertere Begründungen auftreten (Kosko und Zimmerman 2019), aber vertiefende empirische Studien fehlen diesbezüglich insbesondere für jüngere Lernende. Für die Klassen 7-9 liegen Befunde vor, wonach der relative Anteil von Lernenden, die eine mehrschrittige geometrische Beweisaufgabe korrekt lösen können, im Verlauf der Sekundarstufe zunimmt (Ufer 2008). Allerdings wird dieser Befund eher auf eine Entwicklung des Basiswissens zurïckgeführt als auf eine qualitative Entwicklung der Beweiskompetenz und damit nicht unmittelbar auf die Fähigkeit, Verallgemeinerungen vornehmen zu können. Dies würde vermuten lassen, dass mathematisches Argumentieren inhaltsbereichsunabhängige Teilkompetenzen verlangt und auf inhaltsspezifisches mathematisches Wissen als „Anchoring“ (Lithner 2008) zurückgreift.

Quasi-längsschnittliche Studien wie beispielsweise diejenige von Neumann et al. (2014) vermochten für Lernende der Klassen 3 und 4 und zur Kontrastierung von Klasse 6 für Arithmetikaufgaben keine systematischen Leistungsunterschiede zwischen den Jahrgängen nachzuweisen, sondern vielmehr eine Art „Homogenisierung der gezeigten Leistungen im Verlauf der Schulalter" auf einem mittleren Niveau der vierten Klasse (Neumann et al. 2014, S. 121). Auch bei den leistungsfähigsten Lernenden ließ sich in dieser Studie keine Zunahme über die Jahrgangsstufen feststellen. Im Hinblick auf einen ersten Ansatz zur Beschreibung der Entwicklung schriftlichen Argumentierens wäre es interessant zu klären, wie dieselbe Begründungsaufgabe von Schülerinnen und Schülern unterschiedlicher Jahrgangsstufen zu Beginn eines Schuljahres gelöst wird und ob sich in den Bearbeitungen der Aufgaben aus zwei unterschiedlichen Inhaltsbereichen und deren Teilaufgaben Unterschiede zwischen den Jahrgangsstufen finden lassen.

Zusammenfassend lassen sich im Zusammenhang mit schriftlichem Argumentieren zumindest vier Forschungslücken feststellen: 1) Überprüfung des Vorkommens der beschriebenen Teilkompetenzen mathematischen Argumentierens, 2) vergleichende Untersuchungen zum mathematischen Argumentieren in unterschiedlichen Inhaltsbereichen, mit dem Ziel zu klären, ob eher von bereichsspezifischen oder bereichsübergreifenden Teilkompetenzen ausgegangen werden kann, 3) Untersuchungen, die inhaltliche Ideen im Argumentationsprozess in den Blick nehmen, und 4) längsschnittliche oder quasi-längsschnittliche Untersuchungen, die sich mit der Frage möglicher altersabhängiger Entwicklung befassen.

Zur Bearbeitung dieser vier Forschungslücken sind große empirische Studien notwendig, die 1) überprüfen, inwiefern die vier theoretisch hergeleiteten Teilkompetenzen mathematischen Argumentierens (vgl. Abschn. 2.2) schriftlich tatsächlich gezeigt werden, 2) eine vergleichende Betrachtung der Bearbeitung derselben Lernenden von ähnlich aufgebauten Aufgaben in unterschiedlichen Inhaltsbereichen zulassen, 3) die inhaltliche Ideen im Argumentationsprozess im Sinne einer Lösungsbzw. Begründungsidee und damit einer bestimmten Strategie in den Blick nehmen 
und 4) eine längsschnittliche bzw. quasi-längsschnittliche Perspektive einnehmen, um künftig etwaige Entwicklungsverläufe modellieren zu können.

Die vorliegende Studie setzt hier an und stellt anhand von zwei Aufgaben aus zwei unterschiedlichen Inhaltsbereichen - unterteilt in Teilaufgaben entlang der vier beschriebenen Teilkompetenzen - dar, wie diese beiden Aufgaben von einem größeren Sample $(N=866)$ von Lernenden der Jahrgangsstufen 4-6 selbstständig schriftlich bearbeitet werden und welche Lösungs- bzw. Begründungsideen dafür herangezogen werden.

\subsection{Fragestellungen}

Der vorliegende Beitrag bearbeitet folgende drei Fragestellungen, die vor dem Hintergrund der bisherigen Erkenntnisse (Abschn. 2.3) mit entsprechenden Hypothesen ergänzt werden:

1) Welche der vier postulierten Teilkompetenzen zeigen Schülerinnen und Schüler der Jahrgangsstufen 4-6 bei zwei Aufgaben aus zwei unterschiedlichen Inhaltsbereichen?

Gemäß dem aktuellen Forschungsstand (Abschn. 2.3) gehen wir davon aus, dass die Teilkompetenzen Erkennen, Beschreiben/Repräsentieren in der überwiegenden Mehrheit der Fälle korrekt umgesetzt werden können, während die Teilkompetenz Begründen deutlich weniger häufig manifest werden dürfte, aber noch häufiger als das Verallgemeinern. Da es sich beim Begründen und Verallgemeinern um kognitiv sehr anspruchsvolle Vorgänge handelt, vermuten wir, dass diese beiden Handlungen - falls sie überhaupt vollzogen werden - in vielen Fällen nur teilweise korrekt ausgeführt werden.

2) Welche inhaltlichen Ideen entwickeln die Schülerinnen und Schüler beim Beschreiben, Begründen und Verallgemeinern für die Bearbeitung der beiden Aufgaben aus den beiden unterschiedlichen Inhaltsbereichen?

Wir vermuten, dass für das Beschreiben, Begründen und Verallgemeinern in den beiden Inhaltsbereichen nicht dieselben inhaltlichen Ideen in gleicher Häufigkeit herangezogen werden, sondern dass es für einen bestimmten Inhaltsbereich naheliegendere, d.h. offensichtlichere und für Lernende einsichtigere Ideen geben dürfte, die gehäuft auftreten, weil sich die Wissensbasis (,Anchoring“) unterscheidet. Inwiefern dieselbe inhaltliche Idee für die unterschiedlichen Teilkompetenzen verwendet wird, lässt sich aufgrund der bisherigen Erkenntnisse nicht abschätzen. Plausibel scheint, dass eine einmal entwickelte Idee für das Beschreiben, Begründen und Verallgemeinern durchgängig genutzt wird.

3) Lassen sich für die einzelnen Jahrgangsstufen systematische Unterschiede beim Begründen beschreiben und, wenn ja, welche?

In Anbetracht der in Abschn. 2.3 dargestellten kontroversen Befunde zu systematischen Unterschieden zwischen einzelnen Jahrgangsstufen kann zur dritten Fragestellung keine eindeutige Hypothese formuliert werden. Eine plausible Annahme 
ist, dass die Handlungen Begründen und Verallgemeinern mit zunehmendem Alter häufiger erfolgreich ausgeführt werden.

Um die Forschungsfragen bearbeiten und die Hypothesen prüfen zu können, braucht es zum einen entsprechendes Aufgabenmaterial (ähnlicher Aufbau nach Teilaufgaben, zwei unterschiedliche Inhaltsbereiche) und zum anderen ein Analyseinstrument, das die Teilkompetenzen des mathematischen Argumentierens hinsichtlich der Korrektheit und Vollständigkeit ihrer Ausführung sowie der Art der zugrunde liegenden inhaltlichen Idee erfasst. Die Aufgaben müssen sodann von unterschiedlichen Jahrgangsstufen bearbeitet werden können, um Anhaltspunkte bezüglich einer möglichen Entwicklung schriftlichen mathematischen Argumentierens generieren zu können. Diese Ausgangslage wurde in der Studie MaBeLL-INT (Mathematisches Begründen Lehren und Lernen: Intervention) (Brunner 2018) geschaffen, die nachfolgend vorgestellt wird.

\section{Methode}

\subsection{Stichprobe}

An der Studie MaBeLL-INT (Brunner 2018) nahmen insgesamt 54 Klassen der Jahrgangsstufen 4-6 teil. Die Klassen stammten sowohl aus ländlichen als auch aus städtischen Regionen des Ostschweizer Kantons Thurgau. In einzelnen Klassen wurden zusätzlich auch Schülerinnen und Schüler der dritten Jahrgangsstufe $\left(N_{J 3}=54\right)$ unterrichtet, die - den Gegebenheiten der Praxis geschuldet - ebenfalls in die Studie einbezogen wurden. Für die Analysen in diesem Beitrag wurden jedoch lediglich Daten der insgesamt 866 Schülerinnen und Schüler der Jahrgangsstufen 4-6 einbezogen. Davon waren 445 männlich und 421 weiblich. Im Mittel waren die Lernenden

Tab. 1 Übersicht Teilstichproben

\begin{tabular}{|c|c|c|c|}
\hline Personenmerkmal & $\begin{array}{l}\text { Teilstichprobe } 4 \text {. Jahr- } \\
\text { gangsstufe } \\
N_{J 4}=243\end{array}$ & $\begin{array}{l}\text { Teilstichprobe 5. Jahr- } \\
\text { gangsstufe } \\
N_{J 5}=302\end{array}$ & $\begin{array}{l}\text { Teilstichprobe } 6 . \text { Jahr- } \\
\text { gangsstufe } \\
N_{J 6}=321\end{array}$ \\
\hline Geschlecht & $\begin{array}{l}110 \text { weiblich }(45,27 \% \\
\text { des Jahrgangs) } \\
133 \text { männlich } \\
(54,73 \% \text { des Jahr- } \\
\text { gangs) }\end{array}$ & $\begin{array}{l}152 \text { weiblich }(50,33 \% \\
\text { des Jahrgangs) } \\
150 \text { männlich } \\
\text { (49,67\% des Jahr- } \\
\text { gangs) }\end{array}$ & $\begin{array}{l}159 \text { weiblich }(49,53 \% \\
\text { des Jahrgangs) } \\
162 \text { männlich (50,47\% } \\
\text { des Jahrgangs) }\end{array}$ \\
\hline Alter & $\begin{array}{l}\text { Alter }=9,73 \text { Jahre, } \\
S D=0,46\end{array}$ & $\begin{array}{l}\text { Alter }=10,74 \text { Jahre, } \\
S D=0,46\end{array}$ & $\begin{array}{l}\text { Alter }=11,76 \text { Jahre, } \\
S D=0,48\end{array}$ \\
\hline Erstsprache & $\begin{array}{l}197 \text { Deutsch }(81,1 \%) \text {, } \\
46 \text { andere }(18,9 \%)\end{array}$ & $\begin{array}{l}253 \text { Deutsch }(83,8 \%) \text {, } \\
49 \text { andere }(16,2 \%)\end{array}$ & $\begin{array}{l}256 \text { Deutsch }(79,8 \%) \text {, } \\
65 \text { andere }(20,2 \%)\end{array}$ \\
\hline $\begin{array}{l}\text { Mittlere Klassen- } \\
\text { leistung Mathematik } \\
\text { (letzte Zeugnisnote }^{\mathrm{a}} \text { ) }\end{array}$ & Note $=5,27, S D=1,68$ & Note $=5,29, S D=1,81$ & Note $=5,07, S D=1,67$ \\
\hline $\begin{array}{l}\text { Mittlere Klassenleis- } \\
\text { tung Deutsch (letzte } \\
\text { Zeugnisnote) }\end{array}$ & Note $=5,14, S D=1,31$ & Note $=5,09, S D=1,35$ & Note $=5,06, S D=1,35$ \\
\hline
\end{tabular}


10,84 Jahre alt $(S D=0,94)$. Die Größe der Teilstichproben der einzelnen Jahrgangsgruppen ist vergleichbar $\left(N_{J 4}=243, N_{J 5}=302, N_{J 6}=321\right)$; nach Alter und Geschlecht lassen sie sich wie in Tab. 1 aufgeführt charakterisieren.

Bezüglich des Geschlechts unterscheiden sich die drei Teilstichproben nicht signifikant voneinander $\left(\chi^{2}=1,55 ; d f=2 ; p=0,46\right)$. Im Hinblick auf die an der letzten Zeugnisnote gemessenen, auf den Jahrgang bezogenen relativen mittleren sprachlichen $(F=0,236 ; d f=2 ; p=0,790)$ und mathematischen $(F=1,58 ; d f=2 ; p=0,206)$ Voraussetzungen, erweisen sich die drei Teilstichproben ebenfalls als weitestgehend vergleichbar; genauso verhält es sich beim Anteil der Lernenden, deren Erstsprache Deutsch ist $\left(\chi^{2}=1,72 ; d f=2 ; p=0,423\right)$.

\subsection{Aufgabe und Analyseeinheit}

Als Grundlage für die Datenerhebung wurde je eine für die Studie entwickelte Aufgabe $^{5}$ aus den Inhaltsbereichen „Geometrie“ und „Arithmetik“ konzipiert. Jede der beiden Aufgaben umfasst vier Teilaufgaben, die jeweils eine der vier Teilkompetenzen - Erkennen, Beschreiben, Begründen, Verallgemeinern (Abschn. 2.2) adressieren. Dadurch wurden die theoretisch hergeleiteten Handlungen in vier Teilaufgaben getrennt, um diese Teilkompetenzen gezielt herauszufordern und um zu prüfen, ob und wie sie bearbeitet werden. Dadurch soll ermöglicht werden, erfolgreiches mathematisches Argumentieren nicht ausschließlich mit dem Vorliegen des Begründungsschrittes oder des Verallgemeinerns gleichzusetzen, sondern mögliche Teilkompetenzen zu identifizieren.

Beide Aufgaben stellen ein geometrisches bzw. arithmetisches Muster vor und verlangen zunächst das Erkennen (operationalisiert als Weiterführung), bevor in den weiteren Teilaufgaben Beschreiben, Begründen und Verallgemeinern angeregt werden. Der Aufbau der beiden Aufgaben ermöglicht die Vergleichbarkeit der Ausführung der einzelnen Teilkompetenzen in den Teilaufgaben über die beiden unterschiedlichen Inhaltsbereiche hinweg. Inwiefern sich die beiden Aufgaben auch bezüglich des mathematischen Inhalts vergleichen lassen, ist hingegen eine offene Frage, die anhand der verwendeten inhaltlichen Ideen geprüft und anschließend diskutiert werden soll. Die beiden Aufgaben können dem Inhaltsbereich „Muster und Strukturen" (Walther et al. 2008) zugeordnet werden, der im Schweizer Lehrplan (D-EDK 2016) allerdings nicht als eigener Inhaltsbereich ausgewiesen wird, sondern im Fall von Aufgabe A dem Inhaltsbereich „Geometrie“ zugeordnet wird, im Fall von Aufgabe B dem Inhaltsbereich „Arithmetik“.

Aufgabe A (Abb. 1) bezieht sich somit auf den Inhaltsbereich „Geometrie“ und verlangt beim Erkennen eine Fortsetzung des vorgegebenen geometrischen Musters. Danach wird nach einer Beschreibung/Repräsentation des Musters gefragt (,,Schreibe oder zeichne eine Anleitung, wie man das Muster weiterführen kann!"), bevor

\footnotetext{
5 Wir verwenden den Begriff „Aufgabe“ in einer weit gefasst Definition als „Aufforderung zur gezielten Bearbeitung eines eingegrenzten mathematischen Themas" (Neubrand 2002, S. 16) bzw. als Herausforderung zur „Auseinandersetzung mit einer spezifischen mathematischen Situation“ (Neubrand 2002, S. 17) und betrachten Aufgaben damit als ,flexible, breit einsetzbare und aktiv steuerbare inhaltliche und didaktische Strukturierungselemente des Mathematikunterrichts“ sowie als „Träger der kognitiven Aktivitäten“ (Neubrand et al. 2011, S. 116).
} 


\section{Das sind die ersten drei Figuren einer Folge. Zeichne weiter!}

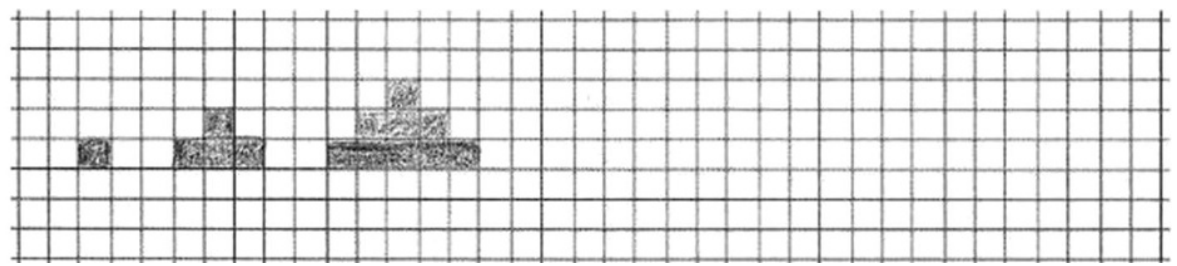

Abb. 1 Aufgabe A, Teilaufgabe 1

anhand des Beispiels der siebten Figur danach gefragt wird, wie viele Kästchen es brauche, um diese Figur zeichnen zu können und warum (,Wie viele Kästchen braucht man für die 7. Figur? Erkläre, warum es genau so viele sind!“"). In Teilaufgabe 4 wird danach gefragt, wie man herausfinden könne, wie viele Kästchen eine beliebige Figur aufweist, und warum dies so sei (,Wie muss deine Erklärung heißen, dass sie immer [für irgendein Beispiel, also auch für die 50., 100. oder 1000. Figur] funktioniert?"“).

Das Pendant zur geometriebezogenen Aufgabe A stellt Aufgabe B für den Inhaltsbereich „Arithmetik“ dar.

In Teilaufgabe 1 wird nach eigenen Beispielen desselben Musters gefragt (Abb. 2), womit ebenfalls das Erkennen der Struktur intendiert wird. Teilaufgabe 2 fordert - auch mit Blick auf die Ergebnisse - dazu auf, das arithmetische Muster zu beschreiben (,Schau dir die Rechnungen und Ergebnisse an. Kannst du ein Muster beschreiben?"). Teilaufgabe 3 verlangt sodann eine Begründung, die sich darauf bezieht, warum aus den drei Zahlen genau dieses Ergebnis resultiert (,Warum entsteht

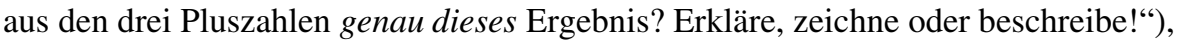

\section{Untersuche die Plusaufgaben und ihre Ergebnisse. Rechne und schreibe zwei weitere Beispiele.}

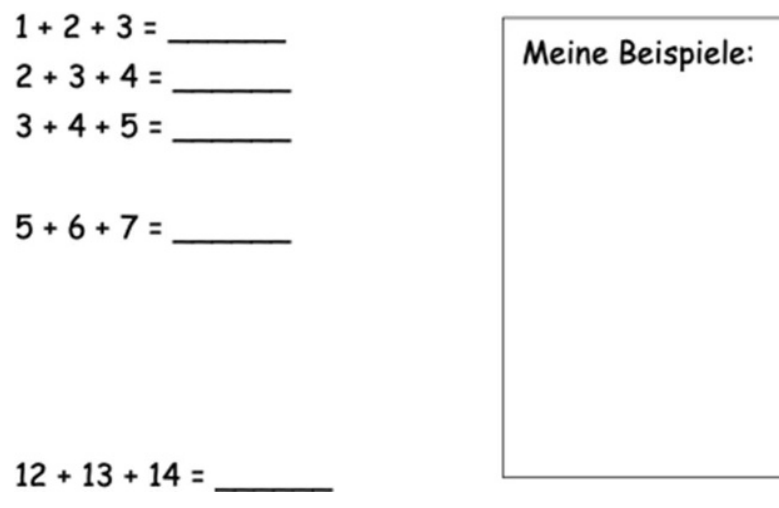

Abb. 2 Aufgabe B, Teilaufgabe 1 
während Teilaufgabe 4 nach einer Verallgemeinerung fragt (,,Wie muss deine Erklärung heißen, damit sie immer funktioniert, also für irgendein Beispiel?“).

Die Bearbeitung der beiden Aufgaben mit ihren je vier Teilaufgaben erfolgte jeweils nach einer kurzen standardisierten mündlichen Einführung schriftlich in zwei unterschiedlichen Unterrichtsstunden an zwei aufeinanderfolgenden Tagen. Begonnen wurde immer mit Aufgabe A, weil diese vom Forschungsteam als einfacher eingeschätzt und deshalb als guter Einstieg betrachtet wurde. Den Lernenden standen je maximal 30 min für die selbstständige schriftliche Bearbeitung zur Verfügung. Die Aufgaben mussten in Einzelarbeit gelöst werden. Als Analyseeinheit gilt nachfolgend die vollständige Bearbeitung der Aufgaben mit ihren vier Teilaufgaben. Dies hat den Vorteil, dass bereits bei Teilaufgabe 2 erfolgte Begründungen oder Verallgemeinerungen ebenfalls erfasst werden.

\subsection{Analyseinstrument}

Für die Studie wurde ein Kodiersystem (Abb. 3) entwickelt, pilotiert und eingesetzt, das verschiedene Bearbeitungsaspekte erfasste. Im Rahmen der vorliegenden Studie wurden folgende analysiert: (a) Teilkompetenzen entlang der vier Handlungen als Teilaufgaben (vgl. Abschn. 2.2), (b) die Ausprägung bzw. Ausführungsqualität der Bearbeitung hinsichtlich Korrektheit und Vollständigkeit sowie (c) die zugrunde liegende inhaltliche Idee (Brunner 2019b; Brunner et al. 2020).

Die einzelnen Kategorien, Subkategorien und Codes wurden in einem ausführlichen Kodierleitfaden festgehalten (Brunner et al. 2019) und entlang der vier Teilkompetenzen - Erkennen, Beschreiben, Begründen, Verallgemeinern - beschrieben. Die Vorgehensweise wurde für jede Teilkompetenz einzeln anhand von Beschreibungen und/oder Ankerbeispielen dargelegt. Die Ankerbeispiele unterscheiden sich für die beiden Aufgaben A und B, die Codes hingegen sind für beide Aufgaben identisch. Diese Vorgehensweise ermöglichte es, die Aufgaben aus zwei unterschiedlichen Inhaltsbereichen vergleichend zu analysieren.

Zunächst wurde für jede Teilkompetenz geprüft, ob sie gezeigt worden war (Antwort - keine Antwort). Lag eine zur Teilaufgabe passende Antwort vor, wurde als Nächstes deren Ausprägung bzw. Ausführungsqualität in Anlehnung an Neumann et al. (2014, S. 118) sowie Kempen (2019) anhand einer ordinalen bzw. metrischen Stufung, die die Korrektheit des vollzogenen Schrittes mit der (inhaltlichen)

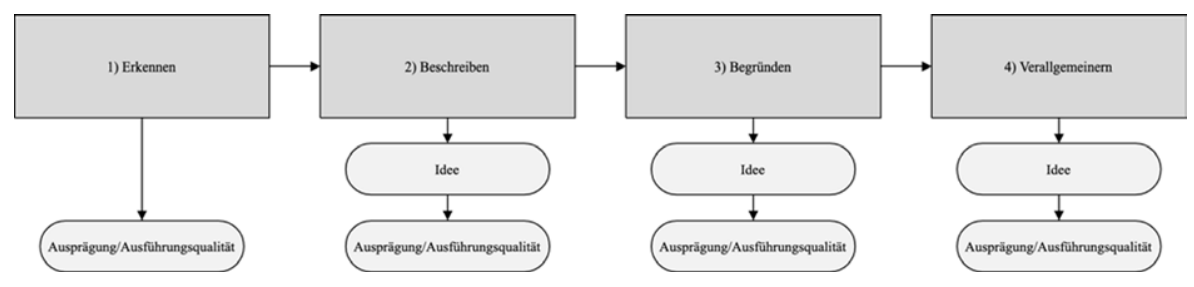

Abb. 3 Ablauf Kodierung entlang der vier Teilprozesse. (Brunner 2019b, S. 1133, Auszug, hier angepasst) 
Tab. 2 Codebeschreibung für Aufgabe A (Auszug): Beschreiben/Repräsentieren - Ausprägung/Ausführungsqualität

\begin{tabular}{|c|c|c|}
\hline $\begin{array}{l}\text { Beschreibung } \\
\text { Code }\end{array}$ & $\begin{array}{l}\text { Ausprägung der } \\
\text { Ausführung }\end{array}$ & Beschreibung \\
\hline \multirow{6}{*}{$\begin{array}{l}\text { Die } \\
\text { erkannte } \\
\text { Struktur } \\
\text { wird be- } \\
\text { schrieben/ } \\
\text { dargestellt } \\
\text { und die } \\
\text { relevanten } \\
\text { Merkmale } \\
\text { werden } \\
\text { herausgear- } \\
\text { beitet }\end{array}$} & 0: nicht korrekt & $\begin{array}{l}\text { Die gegebene Beschreibung ist falsch oder es wird ein völlig } \\
\text { anderer Sachverhalt beschrieben als derjenige, der in der Aufga- } \\
\text { benstellung angelegt ist }\end{array}$ \\
\hline & 1: teilweise korrekt & $\begin{array}{l}\text { Die gegebene Beschreibung enthält inhaltliche (mathematische) } \\
\text { Fehler, ist aber nicht vollständig falsch bzw. enthält korrekte } \\
\text { Teile }\end{array}$ \\
\hline & $\begin{array}{l}\text { 2: korrekt, aber } \\
\text { unvollständig }\end{array}$ & $\begin{array}{l}\text { Die gegebene Beschreibung ist korrekt, es fehlen aber zentrale } \\
\text { Teile der inhaltlichen Idee }\end{array}$ \\
\hline & $\begin{array}{l}\text { 3: korrekt und } \\
\text { vollständig }\end{array}$ & $\begin{array}{l}\text { Die gegebene Beschreibung ist korrekt und die inhaltliche Idee } \\
\text { ist inhaltlich vollständig. Zu Vollständigkeit gehört auch Ein- } \\
\text { deutigkeit, d.h. durch die korrekt gegebene Beschreibung ist } \\
\text { vollständig und eindeutig geklärt, wie die Struktur funktioniert }\end{array}$ \\
\hline & 99: keine Antwort & - \\
\hline & $\begin{array}{l}\text { 97: keine inhalt- } \\
\text { liche, d.h. keine } \\
\text { mathematische } \\
\text { Bearbeitung }\end{array}$ & $\begin{array}{l}\text { Es wurde etwas anderes gemacht als in der Aufgabe verlangt. } \\
\text { Dazu gehören auch Äußerungen/Antworten wie „Kein Bock“, } \\
\text { „Keine Ahnung“, usw. }\end{array}$ \\
\hline
\end{tabular}

Vollständigkeit verband, von 0 bis $3^{6}$ eingeschätzt (Tab. 2). Diese Vorgehensweise ermöglichte es beispielsweise, die Teilkompetenz Beschreiben als „korrekt und vollständig" mit Wert 3 zu erfassen, die Teilkompetenz Begründen dann aber nur noch mit dem Wert 2 für „korrekt, aber unvollständig“.

Wurde bei Teilaufgabe 1 das Muster nicht erwartungsgemäß fortgesetzt, sondern beispielsweise einfach wiederholt, wurde dies als „nicht korrekt“ gewertet (vgl. Tab. 2, Wert 0) und in der Folge auch allfällige Beschreibungen, Begründungen oder Verallgemeinerungen als „keine“ bzw. „,nicht zur Aufgabe passende“ Teilkompetenz gewertet (vgl. Tab. 3, Wert 0).

Für die Teilaufgaben 2-4 (Beschreiben, Begründen, Verallgemeinern) wurden die Antworten auch bezüglich der zugrunde liegenden inhaltlichen Idee analysiert. Auch für die Ideen konnten die Codes für beide Aufgaben identisch gehalten werden. Different sind sie bezüglich der Ankerbeispiele bzw. Beschreibungen. Insgesamt wurden für jede Aufgabe vier verschiedene inhaltliche Ideen sowie eine Restgruppe deduktiv hergeleitet: Anhängen, Ummantelung, Umstrukturierung, besondere Zahlen oder andere Ideen (Tab. 3, Codebeschreibung für Aufgabe A).

Dieselben grundlegenden inhaltlichen Ideen wurden im Kodierleitfaden für Aufgabe B mit entsprechenden Ankerbeispielen und Beschreibungen ausgeführt (Tab. 4). Die Idee „Ummantelung“ wurde bei Aufgabe B beispielsweise dann kodiert, wenn sinngemäß beschrieben worden war, dass jeder Summand um 1 größer werde $(+1)$ als in der Summe vorher und die Summe von drei Summanden somit um 3 größer werde (+3) als die vorherige Summe. Auch für Aufgabe B kann die Idee „Um-

\footnotetext{
${ }^{6}$ Die Handlung „Erkennen“ wurde lediglich mit den Werten 0, 1 oder 2 (0: nicht korrekt; 1: teilweise korrekt; 2: korrekt) erfasst, weil die Differenzierung zwischen „korrekt, aber unvollständig“ und „,korrekt und vollständig" nicht notwendig war.
} 
Tab. 3 Codebeschreibung für Aufgabe A (Auszug): Beschreiben/Repräsentieren - inhaltliche Idee

\begin{tabular}{|c|c|c|}
\hline $\begin{array}{l}\text { Beschreibung } \\
\text { Code }\end{array}$ & $\begin{array}{l}\text { Ausprägung der } \\
\text { Ausführung }\end{array}$ & Beschreibung \\
\hline \multirow{3}{*}{$\begin{array}{l}\text { Die } \\
\text { erkannte } \\
\text { Struktur } \\
\text { wird be- } \\
\text { schrieben/ } \\
\text { dargestellt } \\
\text { und die } \\
\text { relevanten } \\
\text { Merkmale } \\
\text { werden } \\
\text { herausgear- } \\
\text { beitet }\end{array}$} & $\begin{array}{l}\text { 0: keine oder } \\
\text { keine zur Auf- } \\
\text { gabe passende } \\
\text { inhaltliche Idee } \\
\text { erkennbar }\end{array}$ & $\begin{array}{l}\text { Es ist entweder keine inhaltliche Idee erkennbar oder es wird ein } \\
\text { ganz anderer Sachverhalt beschrieben, als er in der Aufgabe angelegt } \\
\text { ist }\end{array}$ \\
\hline & $\begin{array}{l}\text { 1: Anhängen } \\
\text { Boden/Stock/ } \\
\text { Reihe mit } \\
\text { Länge }+2 \\
\text { (Anzahl der } \\
\text { Stufen) }\end{array}$ & $\begin{array}{l}\text { Die grundlegende Idee ist, dass ein weiteres um } 2 \text { Kästchen längeres } \\
\text { „Stockwerk“ (Boden) angehängt wird }\end{array}$ \\
\hline & $\begin{array}{l}\text { 2: Ummante- } \\
\text { lung }\end{array}$ & $\begin{array}{l}\text { Man muss eine weitere Schicht über die ganze Figur legen und } \\
\text { kommt dann zur nächsten. In den Blick genommen wird die Figur } \\
\text { als Ganzes und beschrieben wird der Vorgang, dass man rund herum } \\
\text { eine Schicht über das Ganze anfügt. Zum Beispiel: Man muss in je- } \\
\text { der Reihe auf jeder Seite je eines anhängen und dann noch die Spitze } \\
\text { aufsetzen. }\end{array}$ \\
\hline
\end{tabular}
aufsetzen
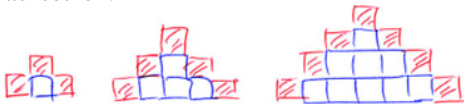

Ummantelung kann zweiseitig (wie Bild) oder einseitig (halbe Figur ummanteln) erfolgen (je 2 auf einer Seite anfügen und in der Mitte zuoberst eines daraufsetzen)

3: Umstruktu-
rierung

4: Besondere

Zahlen
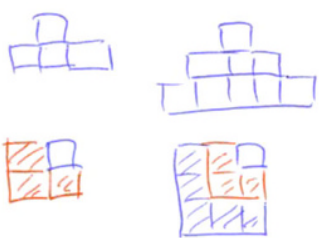

Es wird eine Operation ausgeführt (z. B. Verschieben), mit der der Zusammenhang sofort, d.h. unmittelbar sichtbar wird
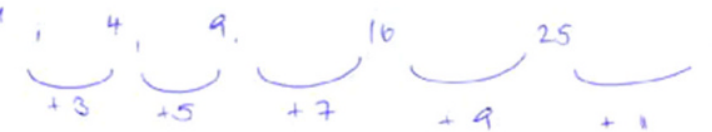

Es wird erkannt, dass es sich immer um Quadratzahlen handelt, d.h., dass Figur $11^{2}$ Kästchen hat, Figur $22^{2}$ Kästchen, Figur $33^{2}$ Kästchen, ... (Verallgemeinerung ist dann Figur $n$ mit $n^{2}$ Kästchen).

Oder: Es wird erkannt, dass die Länge der Stufen immer die Folge der ungeraden Zahlen ist, d. h. zuerst 1 , dann +3 , dann +5 , usw. und somit die Anzahl Kästchen der Summe der entsprechenden Anzahl aufeinanderfolgender ungerader Zahlen entspricht

mantelung“" entsprechend beschrieben werden, wie dies der Kodierleitfaden (Tab. 4) verdeutlicht.

Die Kodierung wurde zunächst in einem Team von drei Personen an zehn Aufgabenblättern erprobt, wonach der Leitfaden konsensbasiert präzisiert wurde. Sodann folgte eine intensive Trainingsphase, in der zwei Kodierende parallel kodierten und 
Tab. 4 Codebeschreibung für Aufgabe B (Auszug): Beschreiben/Repräsentieren - inhaltliche Idee

\begin{tabular}{|c|c|c|}
\hline $\begin{array}{l}\text { Beschreibung } \\
\text { Code }\end{array}$ & $\begin{array}{l}\text { Ausprägung } \\
\text { der Ausfüh- } \\
\text { rung }\end{array}$ & Beschreibung \\
\hline \multirow{4}{*}{$\begin{array}{l}\text { Die } \\
\text { erkannte } \\
\text { Struktur } \\
\text { wird be- } \\
\text { schrieben/ } \\
\text { dargestellt } \\
\text { und die } \\
\text { relevanten } \\
\text { Merkmale } \\
\text { werden } \\
\text { herausgear- } \\
\text { beitet }\end{array}$} & $\begin{array}{l}0 \text { : keine oder } \\
\text { keine zur Auf- } \\
\text { gabe passende } \\
\text { inhaltliche Idee } \\
\text { erkennbar }\end{array}$ & $\begin{array}{l}\text { Es ist entweder keine inhaltliche Idee erkennbar oder es wird ein } \\
\text { ganz anderer Sachverhalt beschrieben, als er in der Aufgabe angelegt } \\
\text { ist }\end{array}$ \\
\hline & 1: Anhängen & $\begin{array}{l}\text { Es wird erkannt, dass es sich um die Summe von drei aufeinander- } \\
\text { folgenden Zahlen handelt. Dabei wird jeweils der erste Summand } \\
\text { weggelassen und durch die nächste Zahl in der Zahlenreihe ersetzt: } \\
1+2+3 \rightarrow+2+3+4\end{array}$ \\
\hline & $\begin{array}{l}\text { 2: Ummante- } \\
\text { lung }\end{array}$ & $\begin{array}{l}\text { Es wird erkannt, dass jeder Summand um } 1 \text { grösser wird }(+1) \text { als in } \\
\text { der Summe vorher und die Summe von drei Summanden somit um } 3 \\
\text { grösser wird }(+3) \text { als die vorherige Summe }\end{array}$ \\
\hline & $\begin{array}{l}\text { 3: Umstruktu- } \\
\text { rierung }\end{array}$ & $\begin{array}{l}\text { Es wird erkannt, dass der erste Summand um } 1 \text { kleiner ist als der } \\
\text { zweite und der dritte um } 1 \text { grösser als der zweite und dass sich die } \\
\text { Differenzen des ersten und dritten Summanden zum mittleren hin } \\
\text { egalisieren und es sich somit um eine Verdreifachung des mittleren } \\
\text { Summanden handelt. (,Es gibt immer dreimal so viel wie die mittlere } \\
\text { Zahl.“) }\end{array}$ \\
\hline- & $\begin{array}{l}\text { 4: Besondere } \\
\text { Zahlen }\end{array}$ & $\begin{array}{l}\text { Es wird erkannt, dass immer die Summe von drei aufeinanderfol- } \\
\text { genden Zahlen gebildet werden muss. Diese ist um } 3 \text { grösser als die } \\
\text { vorhergehende Summe. Hinweis auf diese Idee ist, dass z. B. von } \\
\text { „der Dreierreihe“ gesprochen wird }\end{array}$ \\
\hline
\end{tabular}

die Ergebnisse erneut verglichen und konsensbasiert eine gemeinsame Einschätzung festlegten. Anschließend wurden zweimal zehn Dokumente von den beiden Kodierenden parallel kodiert. Die darauffolgende Überprüfung der Interraterreliabilität fiel gut aus (Cohens Kappa: 0,92 bzw. 0,94, je nach Analysekategorie) (Cohen 1960). Um die große Stichprobe bewältigen zu können, wurden in einem nächsten Schritt insgesamt sechs weitere Kodiererinnen geschult. Nach einer intensiven Trainingsphase wurde erneut auf der Basis von zehn Dokumenten die Interraterreliabilität bestimmt, die für die Gesamtgruppe als zufriedenstellend bis gut bezeichnet werden kann (Fleiss' Kappa: 0,616-0,852, je nach Analysekategorie) (Fleiss 1971). Anschließend wurde die Kodierung von den Kodiererinnen einzeln vorgenommen. Unklare Fälle, bei denen während des Kodierens Unsicherheit aufgetreten war, wurden im Kodierteam besprochen, damit die Kodierung dieser Fälle konsensbasiert definitiv festgelegt werden konnte.

\subsection{Analyse}

Die Daten wurden in SPSS erfasst und bezüglich Konsistenz mehrfach kontrolliert sowie anhand einer Zufallsstichprobe überprüft. Eingesetzt wurden in Abhängigkeit 
von der Fragestellung verschiedene Verfahren deskriptiver Statistik wie Häufigkeitsanalysen, Gruppentests (parametrische und nicht parametrische Verfahren). Für die Bestimmung der Signifikanz von systematischen Unterschieden zwischen den Jahrgängen in den inhaltlichen Ideen (nominale Daten) wurden paarweise $\chi^{2}$-Tests für jeweils zwei Jahrgänge berechnet. Zur Ausführungsqualität wurden die Werte für den Schritt „Begründen“ (Maximum: 3 Punkte; 0: nicht korrekt, 1: teilweise korrekt, 2: korrekt, aber unvollständig, 3: korrekt und vollständig) metrisch interpretiert. Für die Signifikanzbestimmung systematischer Unterschiede zwischen den einzelnen Jahrgängen wurden danach entsprechend parametrische Verfahren (ANOVA, Post-Hoc-Tests nach Bonferroni, $t$-Test) eingesetzt. Die Prüfung von Unterschieden zwischen den beiden Aufgaben innerhalb einer Jahrgangsstufe erfolgte anhand von $t$-Tests für gepaarte Stichproben. Korrelationen wurden zweiseitig nach Pearson berechnet.

Zur Beantwortung der Fragestellungen werden nachfolgend sämtliche Datensätze $(N=866)$ einbezogen. Ausgewiesen werden jeweils Prozentwerte zur Bestimmung der relativen Häufigkeit des Auftretens einer bestimmten inhaltlichen Idee.

\section{Ergebnisse}

Der vorliegende Beitrag konzentriert sich auf drei Fragestellungen (Abschn. 2.4). Die erste Frage zielt auf die auf die Deskription der Bearbeitung der Aufgaben und Teilaufgaben durch die Lernenden der Jahrgangsstufen 4-6 nach Inhaltsbereich ab, während die zweite Frage die Teilaufgaben 2-4 hinsichtlich der verwendeten Ideen und der Korrektheit und Vollständigkeit ihrer Umsetzung in den Blick nimmt. Anhand der dritten Frage soll anschließend nach möglicherweise vorhandenen Unterschieden in den Begründungen der Kinder unterschiedlicher Jahrgangsstufen gesucht werden. Die Ergebnisse zu diesen drei Fragestellungen werden abschnittweise präsentiert.

\subsection{Deskription einzelner Teilkompetenzen mathematischen Argumentierens}

Fragestellung 1 Welche der vier postulierten Teilkompetenzen zeigen Schülerinnen und Schüler der Jahrgangsstufen 4-6 bei zwei Aufgaben aus zwei unterschiedlichen Inhaltsbereichen?

Betrachtet man den Vollzug der einzelnen Handlungen (Abschn. 2.2) dichotom als ausgeführt bzw. nicht ausgeführt (Abb. 4), zeigt sich, dass für beide Aufgaben sämtliche Teilkompetenzen von der überwiegenden Mehrheit der Kinder gezeigt wurden. Die Handlungen Begründen und Verallgemeinern wurden tendenziell etwas seltener vollzogen als die anderen beiden. Am seltensten vollzogen wurden die Verallgemeinerung für beide Aufgaben und das Begründen bei Aufgabe B. Insgesamt liegen Bearbeitungen der vier herausgeforderten Teilkompetenzen für die beiden Aufgaben von 77,02\% (Verallgemeinern, Aufgabe A) bis 97,46\% (Erkennen, Aufgabe A) der Kinder vor.

Betrachtet man die Anzahl nicht gelöster Teilaufgaben/Aufgaben genauer (Tab. 5), zeigt sich für beide Aufgaben deutlich eine zu erwartende Zunahme 


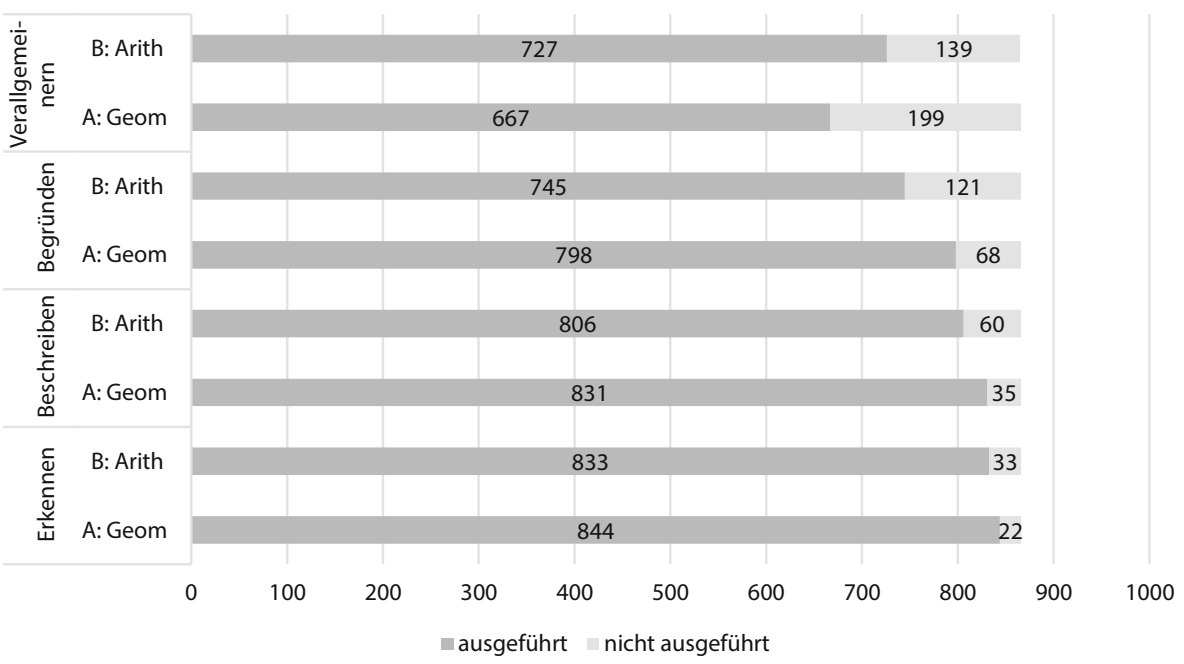

Abb. 4 Vollzug der einzelnen Handlungen (dichotom: ausgeführt - nicht ausgeführt; $N=866$ )

über die vier Teilaufgaben hinweg. Die Häufigkeit, mit der die Kategorie „Teilaufgabe/Aufgabe nicht bearbeitet" kodiert wurde, nimmt über die vier Teilkompetenzen hinweg bei beiden Aufgaben deutlich zu. Dass eine Antwort gegeben wurde, diese aber nicht Bezug nimmt auf die mathematische Anforderung (z. B. „Komme nicht draus"), tritt nach Reihenfolge der Teilaufgaben ebenfalls häufiger auf.

Bezüglich der Korrektheit als Indikator für eine erfolgreiche Bearbeitung zeigt sich für die beiden unterschiedlichen Inhaltsbereiche ein ähnliches Bild (Tab. 6): Während Teilaufgabe 1 überwiegend korrekt und vollständig durchgeführt worden war, fielen die nachfolgenden drei Handlungen den Lernenden zunehmend schwe-

Tab. 5 Art des fehlenden Vollzugs der einzelnen Handlung

\begin{tabular}{|c|c|c|c|c|c|}
\hline $\begin{array}{l}\text { Nicht ausgeführte } \\
\text { Handlung }\end{array}$ & & $\begin{array}{l}\text { Erkennen } \\
\left(N_{A}=22 ;\right. \\
\left.N_{B}=33\right)\end{array}$ & $\begin{array}{l}\text { Beschreiben } \\
\left(N_{A}=35 ;\right. \\
\left.N_{B}=60\right)\end{array}$ & $\begin{array}{l}\text { Begründen } \\
\left(N_{A}=68 ;\right. \\
\left.N_{B}=121\right)\end{array}$ & $\begin{array}{l}\text { Verallgemeinern } \\
\left(N_{A}=199 ;\right. \\
\left.N_{B}=139\right)\end{array}$ \\
\hline \multirow{2}{*}{$\begin{array}{l}\text { a Keine } \\
\text { Verallgemeinerung }\end{array}$} & $\mathrm{A}$ & ${ }^{\mathrm{a}} \mathrm{n} . \mathrm{v}$. & n. v. & n. v. & $17(8,5 \%)$ \\
\hline & B & n. v. & n. v. & n. v. & $0(0,0 \%)$ \\
\hline \multirow{2}{*}{$\begin{array}{l}\text { Teilaufgabe/Aufgabe } \\
\text { nicht bearbeitet }\end{array}$} & A & $20(90,9 \%)$ & $25(71,4 \%)$ & $45(66,2 \%)$ & $118(59,3 \%)$ \\
\hline & B & $25(75,8 \%)$ & $29(48,4 \%)$ & $67(55,4 \%)$ & $73(52,5 \%)$ \\
\hline \multirow{2}{*}{$\begin{array}{l}{ }^{b} \text { Keine inhaltliche, } \\
\text { d.h. mathematische } \\
\text { Bearbeitung }\end{array}$} & A & $2(9,1 \%)$ & $10(28,6 \%)$ & $23(33,8 \%)$ & $64(32,2 \%)$ \\
\hline & B & $8(24,2 \%)$ & $31(51,6 \%)$ & $54(44,6 \%)$ & $66(47,5 \%)$ \\
\hline
\end{tabular}

${ }^{a}$ Verallgemeinern wird nur in Teilaufgabe 4 verlangt. Bei den anderen Teilaufgaben tritt dies nicht auf (n. v.) oder wird ggf. der Teilkompetenz „Verallgemeinern“ zugeordnet. „Keine Verallgemeinerung“ bedeutet daher, dass auf die Herausforderung in Teilaufgabe 4 nicht im Sinne eine Verallgemeinerung reagiert, aber eine mathematische Bearbeitung vorgenommen wurde (z. B. Wiederholung der Beschreibung oder Begründung oder Bearbeitung eines Einzelfalls)

bAntwort, die nicht auf die mathematische Anforderung Bezug nimmt (z. B. „Kein Bock“; „Komme nicht draus.") 
Tab. 6 Leistung Ausprägung/Ausführungsqualität für Aufgaben A und B getrennt (A: Geometrie; $B$ : Arithmetik) in absoluten Werten und gültigen Prozentwerten $(N=866)$

\begin{tabular}{llllll}
\hline $\begin{array}{l}\text { Ausprägung } \\
\text { nach Aufga- } \\
\text { beninhalt }\end{array}$ & $\begin{array}{l}\text { Erkennen } \\
\left(N_{A}=844 ;\right.\end{array}$ & $\begin{array}{l}\text { Beschreiben } \\
\left(N_{A}=831 ;\right.\end{array}$ & $\begin{array}{l}\text { Begründen } \\
\left(N_{A}=798 ;\right.\end{array}$ & $\begin{array}{l}\text { Verallgemeinern } \\
\left(N_{A}=667 ;\right.\end{array}$ \\
\hline Nicht korrekt & A & ${ }^{\mathrm{a}} 144(17,1 \%)$ & $190(22,9 \%)$ & $358(44,9 \%)$ & $308(46,2 \%)$ \\
& $\mathrm{B}$ & ${ }^{\mathrm{a}} 224(26,9 \%)$ & $199(24,7 \%)$ & $427(57,3 \%)$ & $475(65,3 \%)$ \\
Teilweise & $\mathrm{A}$ & $8(0,9 \%)$ & $66(7,9 \%)$ & $135(16,9 \%)$ & $49(7,3 \%)$ \\
korrekt & $\mathrm{B}$ & $111(13,3 \%)$ & $84(10,4 \%)$ & $25(3,4 \%)$ & $26(3,6 \%)$ \\
Korrekt, & $\mathrm{A}$ & $\mathrm{b}$ n. v. & $337(40,6 \%)$ & $153(19,2 \%)$ & $268(40,2 \%)$ \\
unvollständig & $\mathrm{B}$ & $\mathrm{b}$ n.v. & $441(54,7 \%)$ & $257(34,5 \%)$ & $211(29,0 \%)$ \\
Korrekt und & $\mathrm{A}$ & $692(82,0 \%)$ & $238(28,6 \%)$ & $152(19,0 \%)$ & $42(6,3 \%)$ \\
vollständig & $\mathrm{B}$ & $498(59,8 \%)$ & $82(10,2 \%)$ & $36(4,8 \%)$ & $15(2,1 \%)$ \\
\hline
\end{tabular}

ăösungen, die für Teilaufgabe 1 bei Aufgabe A $(N=80)$ oder Aufgabe B $(N=1)$ ein anderes Muster (i.d. R. wurde bei Aufgabe A eine Kopie des Musters vorgenommen oder das Muster gespiegelt) fortsetzen, wurden als ,nicht korrekt“" kodiert

bDie Ausprägung von „Erkennen“" wurde nach ,,nicht korrekt“, ,teilweise korrekt” und „korrekt“ kodiert. Daher ist die Ausprägung „korrekt, aber unvollständig“ bei diesem Schritt nicht anwendbar

rer. Verallgemeinern trat nur relativ selten korrekt oder teilweise korrekt auf. 55,1\% (Teilaufgabe A) bzw. 42,7\% (Teilaufgabe B) der Lernenden, welche die Aufgabe bearbeitet hatten, schafften es hingegen, eine Begründung zu formulieren, die zumindest teilweise korrekt war. Die Anzahl der Lernenden, die eine Teilaufgabe nicht korrekt beantwortet hatten, nahm über die vier Teilaufgaben hinweg wiederum zu. Die Teilkompetenz Erkennen war bei Aufgabe B offenbar schwieriger als die anschließende Beschreibung des Sachverhalts, was bei Aufgabe A nicht der Fall war.

Für beide Aufgaben zeigt sich eine Abnahme der zumindest teilweise korrekten Antworten vom Erkennen über das Beschreiben und Begründen bis zum Verallgemeinern hin.

\subsection{Deskription inhaltliche Ideen}

Fragestellung 2 Welche inhaltlichen Ideen entwickeln die Schülerinnen und Schüler beim Beschreiben, Begründen und Verallgemeinern für die Bearbeitung der beiden Aufgaben aus den beiden unterschiedlichen Inhaltsbereichen?

In den Bearbeitungen beider Aufgaben kommen sämtliche Ideen vor (Tab. 7). Gehäuft trat die Idee „Anhängen“ auf, während „Umstrukturierung“ bei beiden Aufgaben selten feststellbar war. Die Idee „Besondere Zahlen“ wurde bei Aufgabe B (Arithmetik) deutlich häufiger genutzt als bei Aufgabe A (Geometrie). Hingegen trat die „Ummantelung“ bei Aufgabe A deutlich häufiger auf als bei Aufgabe B. Bei Aufgabe A kam es auch häufiger vor als bei Aufgabe B, dass eine andere Idee vorliegt oder sie nicht zugeordnet werden konnte. Die Anzahl der Lernenden, die keine oder keine zur Aufgabe passende inhaltliche Idee entwickelten, nimmt über die drei Teilaufgaben hinweg bei beiden Aufgaben zu, was mit der Abnahme korrekter Bearbeitung korrespondiert. Für Aufgabe A (Geometrie) lässt sich feststellen, dass die Häufigkeit, mit der die Ideen „Anhängen“, „Ummantelung“, ,Umstruktu- 
Tab. 7 Idee für die beiden Aufgaben A und B getrennt ( $A$ : Geometrie; $B$ : Arithmetik) in absoluten Werten und gültigen Prozentwerten $(N=866)$

\begin{tabular}{|c|c|c|c|c|}
\hline Idee nach Aufgabeninhalt & & Beschreiben & Begründen & Verallgemeinern \\
\hline \multirow{2}{*}{$\begin{array}{l}\text { Keine oder keine zur Aufgabe } \\
\text { passende Idee erkennbar }\end{array}$} & $\mathrm{A}$ & $149(17,2 \%)$ & $243(28,1 \%)$ & $253(29,2 \%)$ \\
\hline & B & $184(21,3 \%)$ & $423(48,9 \%)$ & $468(54,0 \%)$ \\
\hline \multirow[t]{2}{*}{ Anhängen } & A & $275(31,8 \%)$ & $168(19,4 \%)$ & $144(16,6 \%)$ \\
\hline & B & $264(30,5 \%)$ & $118(13,6 \%)$ & $121(13,9 \%)$ \\
\hline \multirow[t]{2}{*}{ Ummantelung } & A & $285(32,9 \%)$ & $145(16,7 \%)$ & $147(17,0 \%)$ \\
\hline & $\mathrm{B}$ & $67(7,7 \%)$ & $60(6,9 \%)$ & $47(5,4 \%)$ \\
\hline \multirow[t]{2}{*}{ Umstrukturierung } & A & $2(0,2 \%)$ & $2(0,2 \%)$ & $1(0,1 \%)$ \\
\hline & B & $9(1,0 \%)$ & $13(1,5 \%)$ & $17(2,0 \%)$ \\
\hline \multirow[t]{2}{*}{ Besondere Zahlen } & A & $21(2,4 \%)$ & $60(6,9 \%)$ & $44(5,1 \%)$ \\
\hline & B & $255(29,4 \%)$ & $121(14,0 \%)$ & $62(7,2 \%)$ \\
\hline \multirow[t]{2}{*}{ Andere Idee } & A & $99(11,4 \%)$ & $181(20,9 \%)$ & $97(11,2 \%)$ \\
\hline & B & $27(3,1 \%)$ & $11(1,3 \%)$ & $10(1,2 \%)$ \\
\hline \multirow[t]{2}{*}{ Keine Antwort } & A & $25(2,9 \%)$ & $45(5,3 \%)$ & $118(13,6 \%)$ \\
\hline & B & $29(3,4 \%)$ & $67(7,7 \%)$ & $74(8,6 \%)$ \\
\hline \multirow{2}{*}{$\begin{array}{l}\text { Keine inhaltliche, } d . h . \text { keine } \\
\text { mathematische Bearbeitung }\end{array}$} & A & $10(1,2 \%)$ & $22(2,5 \%)$ & $62(7,2 \%)$ \\
\hline & B & $31(3,6 \%)$ & $53(6,1 \%)$ & $67(7,7 \%)$ \\
\hline
\end{tabular}

Geringfügige Abweichungen (ein Fall bei Begründen und zwei Fälle bei Verallgemeinern Aufgabe A) gegenüber den in Abb. 4 ausgewiesenen Werten ,nicht ausgeführt“ (Ausführungsqualität) kommen durch eine Zuweisung zum Code „keine Idee erkennbar“ zustande, d.h. der Code ,keine inhaltliche, d.h. mathematische Bearbeitung “ bei der Bestimmung der Ausführungsqualität konnte hier hinsichtlich der Idee weiter präzisiert werden (z. B. ,keine Idee“). Fehlende Verallgemeinerungen (Tab. 5) wurden hier ebenfalls diesem präzisierten Code zugewiesen

rierung“ bzw. „Besondere Zahlen“ umgesetzt wurden, bei 154 Lernenden (45,8\% der Lernenden, die sämtliche Schritte bewältigt hatten) über die drei Teilaufgaben hinweg konstant bleibt. Für Aufgabe B (Arithmetik) nutzten 75 Lernende (30,5\%) die gleiche Idee sowohl beim Beschreiben und Begründen als auch beim Verallgemeinern.

\subsection{Unterschiede zwischen den Jahrgangsstufen}

Fragestellung 3 Lassen sich für die einzelnen Jahrgangsstufen systematische Unterschiede beim Begründen beschreiben und, wenn ja, welche?

Zur Beantwortung der dritten Fragestellung wird nachfolgend ausschließlich auf Teilaufgabe 3, das Begründen, fokussiert. Bezüglich der Ausprägung/ Ausführungsqualität bei der Lösung zeigen sich signifikante Unterschiede zwischen den Jahrgangsstufen, und zwar für beide Aufgaben (Tab. 8). Aufgabe A (Geometrie) wurde von den Lernenden der vierten Jahrgangsstufe höchst signifikant schlechter gelöst als von den Lernenden der fünften $(p=0,000)$ und der sechsten Jahrgangsstufe $(p=0,000)$. Der Unterschied zwischen der fünften und der sechsten Jahrgangsstufe hingegen erweist sich nicht als statistisch signifikant. Bei Aufgabe B (Arithmetik) ergibt sich ein Unterschied zwischen den Lernenden der vierten Jahrgangsstufe und den Lernenden der sechsten Jahrgangsstufe $(p=0,004)$, nicht aber zwischen den 
Tab. 8 Ausprägung/Ausführungsqualität der Begründung nach Jahrgangsstufen für die beiden Aufgaben $\mathrm{A}$ und $\mathrm{B}$ getrennt

\begin{tabular}{|c|c|c|c|c|c|}
\hline Merkmal & & $\begin{array}{l}\text { Jahrgangsstufe } 4 \\
\left(N_{A}=205 ;\right. \\
\left.N_{B}=190\right)\end{array}$ & $\begin{array}{l}\text { Jahrgangsstufe } 5 \\
\left(N_{A}=281 ;\right. \\
\left.N_{B}=267\right)\end{array}$ & $\begin{array}{l}\text { Jahrgangsstufe } 6 \\
\left(N_{A}=312 ;\right. \\
\left.N_{B}=288\right)\end{array}$ & $\begin{array}{l}\text { ANOVA (Un- } \\
\text { terschiede } \\
\text { zwischen den } \\
\text { Klassen) }\end{array}$ \\
\hline \multirow[t]{2}{*}{$\begin{array}{l}\text { Ausprägung/ } \\
\text { Ausführungsqualität }\end{array}$} & A & $\begin{array}{l}M=0,70 \\
S D=1,037\end{array}$ & $\begin{array}{l}M=1,25 \\
S D=1,204\end{array}$ & $\begin{array}{l}M=1,29 ; \\
S D=1,177\end{array}$ & $\begin{array}{l}F=19,02 ; d f=2 ; \\
p=0,000\end{array}$ \\
\hline & B & $\begin{array}{l}M=0,69 \\
S D=0,967\end{array}$ & $\begin{array}{l}M=0,84 \\
S D=1,057\end{array}$ & $\begin{array}{l}M=1,01 \\
S D=1,071\end{array}$ & $\begin{array}{l}F=5,58 ; d f=2 \\
p=0,004\end{array}$ \\
\hline $\begin{array}{l}\text { Unterschied in- } \\
\text { nerhalb der Jahr- } \\
\text { gänge } M_{A} \text { und } \\
M_{B}\end{array}$ & - & $\begin{array}{l}t=0,85 \\
d f=174 \\
p=0,398\end{array}$ & $\begin{array}{l}t=4,50 \\
d f=256 \\
p=0,000\end{array}$ & $\begin{array}{l}t=3,51 \\
d f=284 \\
p=0,001\end{array}$ & - \\
\hline
\end{tabular}

Tab. 9 Idee bei der Begründung für die beiden Aufgaben A und B getrennt (A: Geometrie; $B$ : Arithmetik) in absoluten Nennungen und gültigen Prozentwerten

\begin{tabular}{lllll}
\hline Idee & & $\begin{array}{l}\text { Jahrgangsstufe } 4 \\
\left(N_{A}=206 ; N_{B}=191\right)\end{array}$ & $\begin{array}{l}\text { Jahrgangsstufe } 5 \\
\left(N_{A}=281 ; N_{B}=267\right)\end{array}$ & $\begin{array}{l}\text { Jahrgangsstufe 6 } \\
\left(N_{A}=312 ; N_{B}=288\right)\end{array}$ \\
\hline $\begin{array}{l}\text { Keine oder keine zur } \\
\text { Aufgabe passende Idee } \\
\text { erkennbar }\end{array}$ & A & $105(51,0 \%)$ & $75(26,7 \%)$ & $63(20,2 \%)$ \\
Anhängen & B & $123(64,4 \%)$ & $158(59,2 \%)$ & $142(49,3 \%)$ \\
& A & $34(16,5 \%)$ & $61(21,7 \%)$ & $73(23,4 \%)$ \\
Ummantelung & B & $29(15,2 \%)$ & $36(13,5 \%)$ & $53(18,4 \%)$ \\
Umstrukturierung & A & $23(11,2 \%)$ & $44(15,7 \%)$ & $78(25,0 \%)$ \\
& B & $13(6,8 \%)$ & $26(9,7 \%)$ & $21(7,3 \%)$ \\
Besondere Zahlen & A & $0(0,0 \%)$ & $2(0,7 \%)$ & $0(0,0 \%)$ \\
& B & $0(0,0 \%)$ & $5(1,9 \%)$ & $8(2,8 \%)$ \\
Andere Idee & A & $5(2,4 \%)$ & $27(9,6 \%)$ & $28(9,0 \%)$ \\
& B & $26(13,6 \%)$ & $40(15,0 \%)$ & $55(19,1 \%)$ \\
& A & $39(18,9 \%)$ & $72(25,6 \%)$ & $70(22,4 \%)$ \\
& B & $0(0,0 \%)$ & $2(0,7 \%)$ & $9(3,1 \%)$ \\
\hline
\end{tabular}

Schülerinnen und Schülern der vierten und der fünften oder der fünften und der sechsten Jahrgangsstufe.

Fragt man für die einzelnen Jahrgangsstufen nach Unterschieden zwischen der Ausführungsqualität der gegebenen Begründung zwischen Aufgabe A und B (Tab. 8), erweist sich die Ausprägung für die fünfte und die sechste Jahrgangsstufe als statistisch signifikant unterschiedlich, und zwar zugunsten von Aufgabe A. Keinen signifikanten Unterschied in der Ausführungsqualität der beiden Aufgaben gibt es hingegen in der vierten Jahrgangsstufe.

Bezüglich der gewählten Idee (Tab. 9) zeigen sich bei Aufgabe A Unterschiede zwischen der vierten und der fünften $\left(\chi^{2}=35,483 ; d f=5 ; p=0,000\right)$, der vierten und der sechsten $\left(\chi^{2}=60,348 ; d f=4 ; p=0,000\right)$ sowie der fünften und der sechsten $\left(\chi^{2}=12,052 ; d f=5 ; p=0,034\right)$ Jahrgangsstufe. Auch für Aufgabe B lassen sich Unterschiede bei der Wahl der inhaltlichen Idee feststellen, und zwar zwischen der vierten und der sechsten $\left(\chi^{2}=18,779 ; d f=5 ; p=0,002\right)$ und der fünften und der sechs- 
ten Jahrgangsstufe $\left(\chi^{2}=11,369 ; d f=5 ; p=0,045\right)$, nicht aber zwischen der vierten und der fünften Jahrgangsstufe $\left(\chi^{2}=6,998 ; d f=5 ; p=0,221\right)$.

\section{Diskussion}

\subsection{Zusammenfassung der Ergebnisse und Beantwortung der Fragestellungen}

Im vorliegenden Beitrag sollten drei Fragestellungen geklärt werden (Abschn. 2.4). Fragestellung 1 bezog sich auf die Deskription der vier Teilkompetenzen der Lernenden der Jahrgangsstufen 4-6 nach Inhaltsbereich, während anhand von Fragestellung 2 eine Beschreibung der Teilkompetenzen hinsichtlich der verwendeten Ideen sowie der Korrektheit und der Vollständigkeit ihrer Umsetzung vorgenommen werden sollte. Fragestellung 3 schließlich untersuchte möglicherweise vorliegende Unterschiede in den Begründungen der Kinder unterschiedlicher Jahrgangsstufen.

\subsubsection{Fragestellung 1: Deskription Teilkompetenzen.}

Den Ergebnissen zu Fragestellung 1 (Abschn. 4.1) lässt sich entnehmen, dass mehr als $73 \%$ der Lernenden zumindest die ersten beiden Teilaufgaben erfolgreich, d.h. zumindest teilweise korrekt bewältigt hatte und eine beachtlich große Gruppe (mehr als $40 \%$ der Lernenden) auch eine Begründung zumindest teilweise korrekt schriftlich formulieren konnte. Dieser Befund verdeutlicht die Wichtigkeit, Leistungen zum schriftlichen mathematischen Argumentieren von Primarschulkindern nicht nur entlang von erfolgten vollständig korrekten Begründungen zu analysieren, weil somit die Leistungen tendenziell unterschätzt werden dürften. Verallgemeinerungen waren in mehr als 53,8\% (Aufgabe A) bzw. 34,0\% (Aufgabe B) der Fälle häufiger als angenommen korrekt oder teilweise korrekt.

Erwartungskonform ist die Abnahme der erfolgreichen Ausführung über die vier Handlungen hinweg, und zwar bei beiden Aufgaben (Abschn. 2.2). Diese Befunde korrespondieren mit bereits vorhandenen Erkenntnissen, wonach das Formulieren von Begründungen anforderungsreich und fehleranfällig ist (Abschn. 2.3). Zudem kumulieren sich mit der gewählten Anlage von Teilaufgaben für die einzelnen Handlungen Nichtausführungen, d.h. wer nicht begründet (Teilaufgabe 3), kann anschlieBend auch nicht verallgemeinern (Teilaufgabe 4). Was sich hingegen nicht bestätigt hat, ist die Vermutung, dass die kognitiv anspruchsvollen Handlungen Begründen und Verallgemeinern nur teilweise korrekt ausgeführt werden, wenn sie erfolgen.

Die beiden Aufgaben wurden unterschiedlich erfolgreich gelöst (A: 55,1\% zumindest teilweise korrekt, B: 42,7\%). Die Aufgabe aus dem Inhaltsbereich „Arithmetik" scheint demnach deutlich schwieriger zu sein als die aus dem Inhaltsbereich „Geometrie“. Dies könnte damit erklärt werden, dass Aufgabe A mit einem anschaulichen geometrischen Muster arbeitet, während in Aufgabe B ein ähnliches Muster lediglich symbolisch in Form eines Terms repräsentiert wird. Dieser Unterschied in der Repräsentationsform dürfte auch einen Grund dafür darstellen, dass das Erkennen der mathematischen Struktur den Lernenden bei Aufgabe B offensichtlich schwerer fiel als bei Aufgabe A, bei der eine geometrische Struktur vorliegt, die aus 
der Abbildung unmittelbar ablesbar ist (vgl. Duncker 1935; Wertheimer 1964) und die Einsicht erleichtert. Das zugrunde liegende Muster kann bei Aufgabe B, die als Term vorliegt, erst im Rückgriff auf mathematisches Fachwissen erschlossen werden, ist aber nicht unmittelbar aus der Aufgabenstellung ablesbar. Ebenfalls denkbar wäre in diesem Zusammenhang, dass den Kindern aus ihrem Mathematikunterricht Punktemuster deutlich vertrauter sind als das mathematische Handeln und Begründen anhand von Termen und bei Aufgabe A deshalb spezifisches Vorwissen genutzt werden konnte.

Kritisch anzumerken ist, dass die vier Teilkompetenzen in den beiden Aufgaben unterschiedlich operationalisiert wurden.

\subsubsection{Fragestellung 2: Ideen.}

Bei den inhaltlichen Ideen traten bei beiden Aufgaben die Ideen „Anhängen“ und auch „Ummantelung“ relativ häufig auf. Diese Ideen scheinen universeller einsetzbar und naheliegender zu sein. Umstrukturierungen hingegen erfolgten sehr selten und wenn, eher beim arithmetischen Muster (Aufgabe B).

Die Idee „Besondere Zahlen“ wurde relativ selten bei Aufgabe B im Inhaltsbereich „Arithmetik“ verwendet und kaum bei Aufgabe A. Diese Idee scheint sich ebenfalls als abhängig vom Inhaltsbereich und möglicherweise auch von der Repräsentationsform der Aufgabenpräsentation zu erweisen. Dies scheint plausibel zu sein, weil die Idee „Besondere Zahlen“ im Fall der Aufgabe aus dem Inhaltsbereich „Geometrie“ eine umfassendere kognitive Umstrukturierung und höhere kognitive Flexibilität (Spiro und Jehng 1990) voraussetzt, als dies bei der Aufgabe aus dem Inhaltsbereich „Arithmetik“ der Fall ist, bei der die besonderen Zahlen unmittelbar im Ergebnis ablesbar sind und lediglich als solche (Zahlen der „Dreierreihe“) identifiziert werden müssen.

Unsere Hypothesen (Abschn. 2.4), wonach einzelne Ideen vom Inhaltsbereich und/oder von der Aufgabengestaltung abhängig sind, bestätigen sich somit. Inwiefern Kinder dieselbe Idee bei Mustern aus unterschiedlichen Inhaltsbereichen verwenden, müsste vertiefend qualitativ geprüft werden.

Eine einmal eingesetzte Idee wird in der Folge nicht zwingend für die weiteren Handlungen genutzt. Dies war bei Aufgabe A (Geometrie) nur bei 45,8\% der Lernenden, die sämtliche Teilaufgaben bewältigt hatten, und bei Aufgabe B (Arithmetik) nur bei 30,5\% der Lernenden der Fall. Dieser eher erwartungswidrige Befund könnte darauf zurückzuführen sein, dass die Lernenden jede der vier Teilaufgaben wie eine neue Aufgabe bearbeiteten und die Aufgabensegmentierung den Blick auf einen gesamthaften Argumentationsprozess im Sinne einer „Cognitive Unit“ (Boero et al. 1996) versperrte, bei der es Sinn ergibt, eine einmal genutzte Idee für sämtliche Handlungen zu verwenden.

\subsubsection{Fragestellung 3: Unterschiede und Gemeinsamkeiten zwischen den Jahrgangsstufen.}

Unterschiede nach Jahrgangsstufen zeigten sich für das Begründen bei beiden Inhaltsbereichen, für Aufgabe A (Geometrie) deutlich öfter als für Aufgabe B (Arith- 
metik). Die Unterschiede zwischen den Jahrgangsstufen verliefen bezüglich der geprüften Merkmale nicht konsistent, wie dies auch in der Studie von Neumann et al. (2014) der Fall gewesen war. So erzielten die Lernenden aus Jahrgangsstufe 4 bei Aufgabe A schlechtere Leistungen als die Lernenden aus den Jahrgangsstufen 5 und 6, was jedoch bei den Lernenden aus Jahrgangsstufe 5 im Vergleich mit den Schülerinnen und Schülern aus Jahrgangsstufe 6 nicht der Fall war. Bei Aufgabe $B$ unterschieden sich die Leistungen nur zwischen den Jahrgangsstufen 4 und 6 systematisch voneinander. Dies ist deshalb bemerkenswert, weil angesichts des Entwicklungsalters der Kinder in den einzelnen Jahrgangsstufen von einer zunehmend höheren Leistungsfähigkeit hätte ausgegangen werden können, zumal die notwendige mathematische Wissensbasis bei beiden Aufgaben relativ einfach ist. Dennoch ist im Gegensatz zu den Befunden der Studie von Neumann et al. (2014) nicht von einer Homogenisierung der Leistungen auf einem mittleren Niveau des vierten Schuljahres auszugehen, sondern es lässt sich vielmehr eine Leistungszunahme von Jahrgangsstufe $4 \mathrm{zu} 6$ beschreiben, auch wenn sich nicht immer signifikante Unterschiede zur fünften Jahrgangsstufe nachweisen ließen. Bezüglich der verwendeten inhaltlichen Idee zeigten sich bei Aufgabe A Unterschiede zwischen allen Jahrgängen. Für Aufgabe B lagen solche hingegen nur für die Jahrgangsstufen 4 und 6 sowie 5 und 6 , nicht aber 4 und 5 vor. Inwiefern diese Befunde auch mit Klasseneffekten oder mit der gleichzeitig erfolgten Verbreiterung der für das „Anchoring" notwendigen mathematischen Wissensbasis, der einzelnen Jahrgangsstufen zusammenhängen, müsste weiter geprüft werden.

Zusammenfassend kann festgehalten werden, dass sich die Unterschiede zwischen den Jahrgangsstufen nicht ausschließlich auf die Korrektheit der gegebenen Begründung beziehen, sondern auch auf die entwickelte Idee. Dies macht deutlich, dass es zu kurz greift, wenn man mit Blick auf etwaige Unterschiede zwischen einzelnen Jahrgangsstufen lediglich auf die Korrektheit achten würde. Weitere Bearbeitungsmerkmale wie die inhaltliche Idee müssten für eine angemessene Leistungseinschätzung auch einbezogen werden. Gerade das Entwickeln einer kreativen Idee zeugt von vertieftem mathematischem Denken und Einbezug der bereits erworbenen mathematischen Wissensbasis im Sinne des beschriebenen „Anchorings“ (Lithner 2008). Inwiefern es anschließend auch gelingt, diese Idee zu repräsentieren und einen Begründungszusammenhang zu formulieren, ist hingegen eine andere Frage (vgl. Neumann et al. 2014).

Die Ergebnisse zeigen, dass es nicht angemessen ist, Argumentationsleistungen von Lernenden der Primarstufe lediglich hinsichtlich der Korrektheit der Schlussfolgerung zu beurteilen. Dadurch würde man Kinder dieser Altersstufe deutlich unterschätzen, da sich gezeigt hat, dass die große Mehrheit der Kinder die ersten beiden Handlungen erfolgreich ausführen konnte und dass 42,7\% (Aufgabe A) bzw. $55,1 \%$ (Aufgabe B) der Kinder, die die Aufgabe bearbeitet hatten, in der Lage waren, eine zumindest teilweise korrekte Begründung zu formulieren. Das anschließende Verallgemeinern fiel ihnen insgesamt betrachtet dann aber sehr schwer. Allerdings war Verallgemeinern in dieser Altersstufe auch noch nicht zwingend zu erwarten (Abschn. 2.2). 


\subsection{Implikationen für Forschung und Praxis}

Aus der vorliegenden Studie ergeben sich verschiedene Implikationen für Forschung und Praxis. Für die Forschung eröffnen sich durch das entwickelte Kodiersystem, das sich in der Anwendung bezüglich der Interraterreliabilität selbst bei einer großen Gruppe von Kodiererinnen als akzeptabel erwiesen hat, neue Möglichkeiten, insbesondere durch seine Verbindung von Korrektheit und Vollständigkeit mit der inhaltlichen Idee, die Ausdruck eines kreativen Aktes ist und Einblick in die beigezogene Wissensbasis im Sinne eines „Anchorings“ (Lithner 2008) gibt. Für die Praxis könnte das Kodiersystem so weiterentwickelt werden, dass es Lehrpersonen eine präzise Beschreibung des Argumentierprozesses von Lernenden ermöglicht, wodurch sichergestellt werden könnte, dass sich ihre Einschätzung nicht nur auf die Korrektheit der Lösung und die Gültigkeit der Schlussfolgerung bezieht, sondern auch vermehrt beschreibende Aspekte des Bearbeitungsprozesses einbezogen werden und zwischen einzelnen Teilkompetenzen differenziert wird. Ein solches, verstärkt qualitativ orientiertes Vorgehen, das sich mit der Bearbeitung entlang verschiedener Merkmale befasst, würde die kreative Denkleistung der Kinder - manifestiert in der inhaltlichen Idee - angemessener berücksichtigen. Gerade die unterschiedlichen Ideen könnten zudem gewinnbringend für den fachlichen Austausch und die Diskussion in der Klasse über die Plausibilität einer Begründung genutzt werden. Der Blick auf die Ideen könnte schließlich dazu beitragen, das Verständnis für Begründungen auch inhaltlich zu erweitern, ohne dass deswegen die Gültigkeit eines Schlusses oder die Struktur eines Arguments vernachlässigt werden müsste. Dies böte eine Möglichkeit für die unterrichtspraktische Umsetzung der in den Bildungsstandards formulierten Teilkompetenzen ,,mathematische Aussagen hinterfragen und auf Korrektheit prüfen“ sowie „Begründungen ... nachvollziehen“ (Walther et al. 2008, S. 32). Zu diesem Zweck wären ausgearbeitete Lernumgebungen mit reichhaltigen Begründungsaufgaben, die verschiedene Begründungsideen ermöglichen, sinnvoll.

\subsection{Limitationen der Studie und Ausblick}

Eine Limitation der Studie liegt bei den fehlenden Daten zu den individuellen sprachlichen, mathematischen und kognitiven Voraussetzungen der Lernenden, die im Rahmen der Studie aus datenschutzrechtlichen Gründen nicht erhoben werden durften. Auch wenn angesichts der großen Stichprobe nicht von systematischen Verzerrungen ausgegangen werden muss, müssen die vorliegenden Ergebnisse dennoch mit entsprechender Vorsicht interpretiert werden. Es könnte sein, dass sich bei Kontrolle der Intelligenz gewisse Zusammenhänge zwischen den Jahrgängen nicht oder anders zeigen würden. Es kann aber angesichts der Größe der Stichprobe und der Vergleichbarkeit der drei Teilstichproben bezüglich Geschlecht, Erstsprache und letzter Zeugnisnote vermutet werden, dass die Varianz in den drei Jahrgangsgruppen auch hinsichtlich der Intelligenz vergleichbar sein dürfte und die Unterschiede zwischen den Jahrgangsstufen bei der Bearbeitung der Begründungsaufgaben bestehen bleiben dürften. Zu prüfen wären allfällige Unterschiede zwischen den einzelnen Klassen und mögliche Klasseneffekte im Sinne von „Argumentationskulturen“ (Jahnke und Ufer 2015, S. 333) der einzelnen Klassen. 
Die Aufgaben selbst wurden nicht alters- bzw. jahrgangsspezifisch konzipiert, was der Absicht und der Zielsetzung der Studie entspricht. Dennoch erwiesen sie sich in der Bearbeitung - zumindest bezüglich einzelner Aspekte - als alters- bzw. jahrgangssensitiv. Für einen Quasi-Längsschnitt ist dies zwar ein akzeptables Vorgehen. Es kann aber keinen Aufschluss darüber geben, ob der Lösungserfolg mit zunehmendem Alter eher Ausdruck einer breiter verfügbaren mathematischen Wissensbasis ist (vgl. Ufer 2008) und die in den Aufgaben benötigten mathematischen Konzepte deshalb mit zunehmendem Alter als einfacher erscheinen oder ob tatsächlich von einer Entwicklung mathematischen Argumentierens ausgegangen werden kann oder ob lediglich die Fähigkeit, schriftlich zu formulieren, zugenommen hat. Zur Klärung dieser Frage wären elaboriertere statistische Verfahren notwendig, die entsprechende Zusammenhänge modellieren und prüfen. Die Deskription kann da$\mathrm{zu}$ jedoch zumindest einen ersten Beitrag leisten und aufzeigen, welche relevanten Aspekte einbezogen werden sollten.

Offensichtlich haben die beiden Aufgaben zur Nutzung unterschiedlicher inhaltlicher Ideen angeregt. Inwiefern diese Aufgaben- und Inhaltsabhängigkeit der unterschiedlichen Repräsentation der Aufgaben geschuldet ist, müsste weiter vertieft untersucht werden. Studien, die mathematisches Argumentieren in verschiedenen Inhaltsbereichen vergleichend untersuchen, sind daher notwendig, stehen aber bezüglich Aufgabenformulierung vor einigen Herausforderungen. Aufgaben aus unterschiedlichen Inhaltsbereichen müssten sowohl strukturgleich als auch sprachlich und bezüglich Repräsentationsform vergleichbar gestaltet sein und darüber hinaus vergleichbare inhaltliche Ideen identifizieren, die zur Problemlösung genutzt werden können. Im Hinblick auf diese Lösungs- oder Begründungsideen könnte zudem die Frage vertieft untersucht werden, inwiefern es sich dabei möglicherweise um inhaltsübergreifende Begründungsideen und damit eigentliche Strategien handelt.

Eine weitere Frage betrifft die Operationalisierung des „Erkennens“. Hier wurden die Leistungen derjenigen 80 Kinder, die das vorhandene Muster bei Aufgabe A (Geometrie) nicht im erwarteten Sinn weitergeführt hatten, wie auch die Leistung desjenigen Kindes, bei dem dies bei Aufgabe B (Arithmetik) nicht der Fall gewesen war, als „nicht korrekt“ kodiert. Es könnte jedoch sein, dass diese Kinder ihr nicht erwartungskonform umgesetztes Muster konsistent beschrieben, begründet und verallgemeinert hatten und in der Studie deshalb unterschätzt wurden. Dies müsste zumindest für die 80 Fälle bei Aufgabe A genauer überprüft werden.

Im Zusammenhang mit der erfassten Korrektheit wäre - zusammen mit anderen Studien - breiter zu diskutieren, inwiefern sich teilweise korrekte Antworten auf einem Kontinuum metrisch interpretieren lassen.

Als Basis für das entwickelte Analyseinstrument dienten die vier in Abschn. 2.2 postulierten Teilkompetenzen mathematischen Argumentierens, die anschließend in vier Teilaufgaben operationalisiert wurden, die die Aufgaben strukturieren. Es war deshalb erwartbar, dass die vier Handlungen von den Kindern auch tatsächlich vollzogen wurden. $\mathrm{Ob}$ dies auch für eine Aufgabe gelten würde, die nicht entlang der vier Teilkompetenzen in Teilaufgaben strukturiert ist, müsste entsprechend überprüft werden. Zudem müsste die Abhängigkeit zwischen den einzelnen Teilkompetenzen weiter untersucht werden. 
Das entwickelte reliable Kodiersystem hat sich bewährt, um Aufgaben aus unterschiedlichen Inhaltsbereichen bezüglich relevanter Bearbeitungsmerkmale des Lösungsprozesses sowie der genutzten inhaltlichen „Idee“ analysieren zu können. Dass dieselben Ideen bei Aufgaben aus unterschiedlichen Inhaltsbereichen beschrieben werden konnten, dürfte auch damit zusammenhängen, dass beide Aufgaben auf das Erkennen und Begründen eines Musters abzielen.

Funding Open access funding provided by Thurgau University of Teacher Education

Open Access Dieser Artikel wird unter der Creative Commons Namensnennung 4.0 International Lizenz veröffentlicht, welche die Nutzung, Vervielfältigung, Bearbeitung, Verbreitung und Wiedergabe in jeglichem Medium und Format erlaubt, sofern Sie den/die ursprünglichen Autor(en) und die Quelle ordnungsgemäß nennen, einen Link zur Creative Commons Lizenz beifügen und angeben, ob Änderungen vorgenommen wurden.

Die in diesem Artikel enthaltenen Bilder und sonstiges Drittmaterial unterliegen ebenfalls der genannten Creative Commons Lizenz, sofern sich aus der Abbildungslegende nichts anderes ergibt. Sofern das betreffende Material nicht unter der genannten Creative Commons Lizenz steht und die betreffende Handlung nicht nach gesetzlichen Vorschriften erlaubt ist, ist für die oben aufgeführten Weiterverwendungen des Materials die Einwilligung des jeweiligen Rechteinhabers einzuholen.

Weitere Details zur Lizenz entnehmen Sie bitte der Lizenzinformation auf http://creativecommons.org/ licenses/by/4.0/deed.de.

\section{Literatur}

Balacheff, N. (1988). Aspects of proof in pupils' practice of school mathematics. Mathematics, teachers and children, 216, 235.

Bezold, A. (2009). Förderung von Argumentationskompetenzen durch selbstdifferenzierende Lernangebote: Eine Studie im Mathematikunterricht der Grundschule. Hamburg: Kovač.

Blum, W., \& Kirsch, A. (1991). Preformal proving: examples and reflections. Educational Studies in Mathematics, 22(2), 183-203.

Boero, P., Douek, N., Morselli, F., \& Pedemonte, B. (2010). Argumentation and proof: A contribution to theoretical perspectives and their classroom implementation. In M. M.F. Pinto \& T.F. Kawasaki (Hrsg.), Proceeding of the 34th Conference of the International Group for the Psychology of Mathematics Education (Bd. 1, S. 179-204). Belo Horizonte: PME.

Boero, P., Garuti, R., Lemut, E., \& Mariotti, A. M. (1996). Challenging the traditional school approach to theorems: a hypothesis about the cognitive unit of theorems. In L. Puig \& A. Gutierrez (Hrsg.), Proceedings of PME 29 (Bd. 2, S. 113-120). Valencia: University of Valencia.

Brunner, E. (2013). Innermathematisches Beweisen und Argumentieren in der Sekundarstufe I. Münster: Waxmann.

Brunner, E. (2014). Mathematisches Argumentieren, Begründen und Beweisen: Grundlagen, Befunde und Konzepte. Heidelberg: Springer.

Brunner, E. (2018). Mathematisches Begründen Lehren und Lernen: Intervention (MaBeLL-INT). Projektbeschreibung. Kreuzlingen: PH Thurgau.

Brunner, E. (2019a). Förderung mathematischen Argumentierens im Kindergarten: Erste Erkenntnisse aus einer Pilotstudie. Journal für Mathematik-Didaktik, 40(2), 323-356.

Brunner, E. (2019b). Wie lassen sich schriftliche Begründungen von Schülerinnen und Schülern des 5. und 6. Schuljahrs beschreiben? In S. Krauss, K. Binder \& A. Frank (Hrsg.), Beiträge zum Mathematikunterricht 2019 (S. 1131-1134). Münster: VTM.

Brunner, E., Jullier, R., \& Lampart, J. (2019). Kodierleitfaden MaBeLL-INT: Aufgabenbearbeitungen t1 a. Kreuzlingen: PHTG.

Brunner, E., Lampart, J., \& Jullier, J. (2020). Rekonstruktion des Argumentationsprozesses anhand schriftlicher Begründungen von Schülerinnen und Schüler. In H.-S. Siller, W. Weigel \& J. F. Wörler (Hrsg.), Beiträge zum Mathematikunterricht 2020 (S. 189-192). Münster: WTM.

Cohen, J. (1960). A coefficient for agreement for nominal scales. Education and Psychological Measurement, (20), 37-46. https://doi.org/10.1177/001316446002000104 
D-EDK (Hrsg.). (2016). Lehrplan 21. Mathematik. www.lehrplan21.ch. Gesehen 24. März 2020

Duncker, K. (1935). Zur Psychologie des produktiven Denkens. Berlin: Springer.

Durand-Guerrier, V. (2008). Truth versus validity in mathematical proof. ZDM Mathematics Education, 40(3), 373-384.

Duval, R. (1991). Structure du raisonnement déductive et apprentissage de la démonstration. Educational Studies in Mathematics, 22(3), 233-261.

English, L. D. (2004). Mathematical and analogical reasoning in early childhood. In L. D. English (Hrsg.), Mathematical and analogical reasoning of young learners (S. 1-22). New York: Routledge.

Fleiss, J.L. (1971). Measuring nominal scale agreement among many raters. Psychological Bulletin, 76(5), 378-382.

Galotti, K. M., Komatsu, L. K., \& Voelz, S. (1997). Children's differential performance on deductive and inductive syllogisms. Developmental Psychology, 33(1), 70-78.

Goldberg, E. (1988). Dürfen Schüler beim Beweisen im Mathematikunterricht so reden, ,wie ihnen der Schnabel gewachsen ist"? Mathematik in der Schule, 16(9), 593-600.

Hanna, G. (1997). The ongoing value of proof. Journal für Mathematikdidaktik, 18(2/3), 171-185.

Heinze, A. (2004). Schülerprobleme beim Lösen von geometrischen Beweisaufgaben - Eine Interventionsstudie. ZDM Mathematics Education, 36(5), 150-161.

Heinze, A., \& Reiss, K. (2004). The teaching of proof at lower secondary level—a video study. ZDM Mathematics Education, 36(3), 98-104.

IQB (2013). Beispielsaufgaben Mathematik Primarstufe. https://www.iqb.hu-berlin.de/vera/aufgaben/ map. Zugegriffen: 15. Apr. 2020.

Jablonski, S., \& Ludwig, M. (2019). Kodierung und Analyse von mündlichen Argumentationsprodukten mithilfe des Toulmin-Schemas. In A. Frank, S. Krauss \& K. Binder (Hrsg.), Beiträge zum Mathematikunterricht 2019 (S. 1139-1142). Münster: WTM.

Jahnke, H. N., \& Ufer, S. (2015). Argumentieren und Beweisen. In R. Bruder, L. Hefendehl-Hebeker, B. Schmidt-Thieme \& H.-G. Weigand (Hrsg.), Handbuch der Mathematikdidaktik (S. 331-355). Heidelberg: Springer Spektrum.

Jeannotte, D., \& Kieran, C. (2017). A conceptual model of mathematical reasoning for school mathematics. Educational Studies in Mathematics, 96(1), 1-16.

Kempen, L. (2013). Generische Beweise in der Hochschullehre. In G. Greefrath, F. Käpnick \& M. Stein (Hrsg.), Beiträge zum Mathematikunterricht 2013 (Bd. 1, S. 528-531). Münster: VTM.

Kempen, L. (2019). Begründen und Beweisen im Übergang von der Schule zur Hochschule: Theoretische Begründung, Weiterentwicklung und Evaluation einer universitären Erstsemesterveranstaltung unter der Perspektive der doppelten Diskontinuität. Wiesbaden: Springer.

KMK (2005). Bildungsstandards im Fach Mathematik für den Primarbereich (Jahrgangsstufe 4). München: Wolters. https://www.kmk.org/fileadmin/Dateien/veroeffentlichungen_beschluesse/2004/2004_10_ 15-Bildungsstandards-Mathe-Primar.pdf. Zugegriffen: 2. Mai 2020.

KMK, \& IQB (2017). Kompetenzstufenmodell zu den Bildungsstandards im Fach Mathematik für den Primarbereich (Jahrgangsstufe 4). https://www.iqb.hu-berlin.de/bista/ksm. Zugegriffen: 2. Mai 2020.

Kosko, K. W., \& Zimmerman, B.S. (2019). Emergence of argument in children's mathematical writing. Journal of Early Childhood Literacy, 19(1), 82-106.

Krauthausen, G. (2001). Wann fängt das Beweisen an? Jedenfalls, ehe es einen Namen hat. Zum Image einer fundamentalen Tätigkeit. In W. Wieser \& B. Wollring (Hrsg.), Beiträge zur Didaktik der Mathematik für die Primarstufe. Festschrift für Siegbert Schmidt (S. 99-113). Hamburg: Kovač.

Krummheuer, G. (2007). Argumentation and participation in the primary mathematics classroom: two episodes and related theoretical abductions. Journal of Mathematical Behavior, 26(1), 60-82.

Krumsdorf, J. (2009). Beispielgebundenes Beweisen. In M. Neubrand (Hrsg.), Beiträge zum Mathematikunterricht 2009 (S. 711-714). Münster: WTM.

Landgraf, V. (2019). Die Pilotstudie des Projekts ,Anschauliches Beweisen im Mathematikunterricht der Grundschule' (schauMal). In A. Frank, S. Krauss \& K. Binder (Hrsg.), Beiträge zum Mathematikunterricht 2019 (S. 469-472). Münster: WTM.

Leiss, D., \& Blum, W. (2006). Beschreibung zentraler mathematischer Kompetenzen. In W. Blum, C. Drüke-Noe, R. Hartung \& O. Köller (Hrsg.), Bildungsstandards Mathematik: Konkret. Sekundarstufe I: Aufgabenbeispiele, Unterrichtsanregungen, Fortbildungsideen (S. 33-50). Berlin: Cornelsen.

Lindmeier, A., Brunner, E., \& Grüssing, M. (2018). Early mathematical reasoning-theoretical foundations and possible assessment. In E. Bergqvist, M. Österholm, C. Granberg \& L. Sumpter (Hrsg.), Proceedings of the 42nd conference of the international group for the psychology of mathematics education (Bd. 3, S. 315-322). Umea: University. 
Lithner, J. (2008). A research framework for creative and imitative reasoning. Educational Studies in Mathematics, 67(3), 255-276.

Mason, J., \& Pimm, D. (1984). Generic examples: seeing the general in the particular. Educational Studies in Mathematics, 15, 277-289.

Neubrand, J. (2002). Eine Klassifikation mathematischer Aufgaben zur Analyse von Unterrichtssituationen: Selbsttätiges Arbeiten in Schülerarbeitsphasen in den Stunden der TIMSS-Video-Studie. Hildesheim: Franzbecker.

Neubrand, M., Jordan, A., Krauss, S., Blum, W., \& Löwen, K. (2011). Aufgaben im COACTIV-Projekt: Einblicke in das Potenzial für kognitive Aktivierung im Mathematikunterricht. In M. Kunter, J. Baumert, W. Blum, U. Klusmann, S. Krauss \& M. Neubrand (Hrsg.), Professionelle Kompetenz von Lehrkräften. Ergebnisse des Forschungsprogramms COACTIV (S. 115-132). Münster: Waxmann.

Neumann, A., Beier, F., \& Ruwisch, S. (2014). Schriftliches Begründen im Mathematikunterricht. Zeitschrift für Grundschulforschung, 2014(1), 113-125.

Pedemonte, B. (2007). How can the relationship between argumentation and proof be analysed? Educational Studies in Mathematics, 66, 23-41.

Polya, G. (1954). Induktion und Analogie in der Mathematik. Mathematik und plausibles Schliessen, Bd. 1. Basel: Birkhäuser.

Reid, D. A., \& Knipping, C. (2010). Proof in mathematics education. Research, learning and teaching. Rotterdam: Sense Publishers.

Reiss, K., Heinze, A., Kuntze, S., Kessler, S., Rudolph-Albert, F., Renkl, A., \& Allolio-Näcke, L. (2006). Mathematiklernen mit heuristischen Lösungsbeispielen. In M. Prenzel (Hrsg.), Untersuchungen zur Bildungsqualität von Schule - Abschlussbericht des DFG-Schwerpunktprogramms (S. 194-210). Münster: Waxmann.

Schmid, S. (2017). Niveaustufen bei impliziten und expliziten Aufgabenformaten geometrischen Begründens. In U. Kortenkamp \& A. Kuzle (Hrsg.), Beiträge zum Mathematikunterricht 2017 (S. 1423-1424). Münster: WTM.

Schwarzkopf, R. (2000). Argumentationsprozesse im Mathematikunterricht. Theoretische Grundlagen und Fallstudien. Hildesheim: Franzbecker.

Schwarzkopf, R. (2015). Argumentationsprozesse im Mathematikunterricht der Grundschule: Ein Einblick. In A. Budke, M. Kuckuck, M. Meyer, F. Schäbitz, K. Schlüter \& G. Weiss (Hrsg.), Fachlich argumentieren lernen. Didaktische Forschungen zur Argumentation in den Unterrichtsfächern (S. 31-45). Münster: Waxmann.

Spiro, R. J., \& Jehng, J.C. (1990). Cognitive flexibility and hypertext: theory and technology for the nonlinear and multidimensional traversal of complex subject matter. In D. Nix \& R. Spiro (Hrsg.), Cognition, education, and multimedia: Exploring ideas in high technology (S. 163-205). Hillsdale: Lawrence Erlbaum.

Stylianides, A.J. (2016). Proving in the elementary mathematics classroom. Oxford: Oxford University Press.

Sumpter, L. (2018). Grade 9 students' reasoning about division of fractions: What are their arguments anchored in? In H. Palmér \& J. Skott (Hrsg.), Students' and teachers' values, attitudes, feelings and beliefs in mathematics classrooms. Selected papers from the 22nd MAVI Conference (S. 135-144). Cham: Springer.

Tebaartz, P.C., \& Lengnink, K. (2015). Was heißt „mathematischer Beweis“? - Realisierungen in Schülerdokumenten. In A. Budke, M. Kuckuck, M. Meyer, F. Schäbitz, K. Schlüter \& G. Weiss (Hrsg.), Fachlich argumentieren lernen. Didaktische Forschungen zur Argumentation in den Unterrichtsfächern (S. 105-122). Münster: Waxmann.

Toulmin, S. E. (1996). Der Gebrauch von Argumenten (2. Aufl.). Weinheim: Beltz.

Ufer, S. (2008). Entwicklung geometrischer Beweiskompetenz in der Sekundarstufe I. In E. Vasarhelyi (Hrsg.), Beiträge zum Mathematikunterricht 2008 (S. 771-774). Münster: WTM.

Ufer, S., Heinze, A., Kuntze, S., \& Rudolph-Albert, F. (2009). Beweisen und Begründen im Mathematikunterricht. Die Rolle von Methodenwissen für das Beweisen in der Geometrie. Journal für MathematikDidaktik, 30(1), 30-54.

Unterhauser, E., \& Gasteiger, H. (2017). „Das ist ein Viereck, weil das hat 4 Ecken.“ - Begründungen von Kindergartenkindern bei Identifikationsentscheidungen für die Begriffe Viereck und Dreieck. In U. Kortenkamp \& A. Kuzle (Hrsg.), Beiträge zum Mathematikunterricht 2017 (S. 981-984). Münster: WTM.

Vogt, M. (2021). Schriftliche Begründungskompetenzen in stochastischen Aufgabenkontexten: Eine Evaluations- und Interventionsstudie im 4. Schuljahr. Wiesbaden: Springer. 
Walther, G., Selter, C., \& Neubrand, J. (2008). Die Bildungsstandards Mathematik. In G. Walther, M. van den Heuvel-Panhuizen, D. Granzer \& O. Köller (Hrsg.), Bildungsstandards für die Grundschule: Mathematik konkret (S. 16-41). Berlin: Cornelsen.

Welsing, F. (2017). Argumentationsprozesse beim Verallgemeinern anschaulich dargestellter arithmetischer Gesetzmäßigkeiten. In U. Kortenkamp \& A. Kuzle (Hrsg.), Beiträge zum Mathematikunterricht 2017 (S. 1017-1020). Münster: WTM.

Wertheimer, M. (1964). Produktives Denken (2. Aufl.). Frankfurt: Kramer.

Wittmann, E. C., \& Müller, N. G. (1988). Wann ist ein Beweis ein Beweis? In P. Bender (Hrsg.), Mathematikdidaktik - Theorie und Praxis. Festschrift für Heinrich Winter (S. 237-258). Berlin: Cornelsen. 